\title{
Current Advances towards 4-Hydroxybutyrate Containing Polyhydroxyalkanoates Production for Biomedical Applications
}

\author{
Ruchira Mitra ${ }^{1,2}$, Hua Xiang ${ }^{1,3, *}$ and Jing Han $1,3, *$ (D) \\ 1 State Key Laboratory of Microbial Resources, Institute of Microbiology, Chinese Academy of Sciences, \\ Beijing 100101, China; mitra.ruchira@yahoo.com \\ 2 International College, University of Chinese Academy of Sciences, Beijing 100049, China \\ 3 College of Life Science, University of Chinese Academy of Sciences, Beijing 100049, China \\ * Correspondence: xiangh@im.ac.cn (H.X.); hanjing@im.ac.cn (J.H.)
}

Citation: Mitra, R.; Xiang, H.; Han, J. Current Advances towards

4-Hydroxybutyrate Containing

Polyhydroxyalkanoates Production for Biomedical Applications.

Molecules 2021, 26, 7244. https://

doi.org/10.3390/molecules26237244

Academic Editors: Ishwar Singh, David Barker and Tao Chen

Received: 31 October 2021

Accepted: 27 November 2021

Published: 29 November 2021

Publisher's Note: MDPI stays neutral with regard to jurisdictional claims in published maps and institutional affiliations.

Copyright: (c) 2021 by the authors. Licensee MDPI, Basel, Switzerland. This article is an open access article distributed under the terms and conditions of the Creative Commons Attribution (CC BY) license (https:// creativecommons.org/licenses/by/ $4.0 /)$.

\begin{abstract}
Polyhydroxyalkanoates (PHA) are polyesters having high promise in biomedical applications. Among different types of PHA, poly-4-hydroxybutyrate $(\mathrm{P} 4 \mathrm{HB})$ is the only polymer that has received FDA approval for medical applications. However, most PHA producing microorganisms lack the ability to synthesize P4HB or PHA comprising 4-hydroxybutyrate $(4 \mathrm{HB})$ monomer due to their absence of a $4 \mathrm{HB}$ monomer supplying pathway. Thus, most microorganisms require supplementation of $4 \mathrm{HB}$ precursors to synthesize $4 \mathrm{HB}$ polymers. However, usage of $4 \mathrm{HB}$ precursors incurs additional production cost. Therefore, researchers have adopted strategies to reduce the cost, such as utilizing low-cost substrate as well as constructing $4 \mathrm{HB}$ monomer supplying pathways in microorganisms. We herein summarize the biomedical applications of $\mathrm{P} 4 \mathrm{HB}$, the natural producers of $4 \mathrm{HB}$ polymer, and the various strategies that have been applied in producing $4 \mathrm{HB}$ polymers in non- $4 \mathrm{HB}$ producing microorganisms. It is expected that the readers would gain a vivid idea on the different strategic developments in the field of $4 \mathrm{HB}$ polymer production.
\end{abstract}

Keywords: microbial synthesis; polyhydroxyalkanoates; 4HB-containing PHA; elastic polymer

\section{Introduction}

As the global concerns about environmental sustainability is rising, bioplastics are gaining increasing importance as a viable alternative to petrochemical-based plastics [1] Bioplastics are environmentally friendly, biodegradable materials derived from renewable sources. Polyhydroxyalkanoates (PHA) are polyesters usually produced by various bacteria and archaea as carbon and energy storage under unbalanced growth conditions of limiting nutrients and excess carbon supply [2]. Besides complete biodegradability in nature, the mechanical, crystal, and thermal properties of PHA are almost comparable with the petroleum counterparts [3]. Importantly, properties of PHA polymers somehow depend on their monomer composition. By changing the monomer type and composition, PHA with favorable material properties can be achieved [4]. There are at least 150 different hydroxyalkanoates that have been identified as PHA monomers [5]. Depending upon the carbon length of these monomers, PHA are classified as short-chain length (SCL-PHA, C3-C5 monomer), medium-chain length (MCL-PHA, C6-C14 monomer), and long-chain length (LCL-PHA, more than 14 carbons in monomer) [6].

SCL-PHA are the most commonly synthesized polymers by bacteria and archaea. Three monomers of SCL-PHA that have received considerable attention are 3-hydroxybutyrate (3HB), 3-hydroxyvalerate (3HV), and 4-hydroxybutyrate (4HB). Polyhydroxybutyrate (PHB), the homopolymer of $3 \mathrm{HB}$, is the most widely produced PHA. PHB is brittle and highly crystalline in nature [7]. It has a high melting point and narrow processing window that makes its industrial processing difficult [8]. Interestingly, the copolymer of $3 \mathrm{HB}$ and $3 \mathrm{HV}$, poly(3-hydroxybutyrate-co-3-hydroxyvalerate) (PHBV) shows enhanced mechanical properties like tensile strength, toughness, and Young's modulus, and also reduced melting 
point [9]. The polymer becomes less crystalline that improves its processing ability. Notably, the homopolymer of $4 \mathrm{HB}$, poly-4-hydroxybutyrate $(\mathrm{P} 4 \mathrm{HB})$, features superior properties and it is the only PHA that has received FDA approval for medical applications since 2007 [10].

Microbial synthesis of $4 \mathrm{HB}$-containing PHA is one of the hot topics in PHA field. Researchers are continuously engrossed in developing various strategies to realize $\mathrm{P} 4 \mathrm{HB}$ synthesis in microbes, and to achieve $4 \mathrm{HB}$-containing polymers with tailor-made $4 \mathrm{HB}$ content. The approaches range from precursor supplementation to metabolic engineering of microbial strains. The present review discusses the various research techniques of $\mathrm{P} 4 \mathrm{HB}$ or its copolymer synthesis. Readers shall gain an understanding as to how the techniques have evolved to enhance the production of the polymer. In this review, we have first discussed the medical applications of P4HB. In the next section, we have presented the natural producers of $4 \mathrm{HB}$ polymer and also the $4 \mathrm{HB}$ synthesis pathway. Following this, we have categorically divided the different approaches of obtaining $4 \mathrm{HB}$ polymers in nonnatural $4 \mathrm{HB}$ producers. These include precursor supplementation, utilization of low-cost substrates, optimization of cultivation parameters, and finally metabolic engineering to construct $4 \mathrm{HB}$ synthesis pathways.

\section{Medical Application of P4HB}

$\mathrm{P} 4 \mathrm{HB}$ is a strong, pliable, and resorbable thermoplastic biomaterial [11]. P4HB is extremely elastic, and its tensile properties are almost comparable to the ultra-high-molecularweight polyethylene [12]. It is highly ductile in nature and has an elongation to break of $1000 \%$, indicating that it can be stretched ten times to its original size before breaking [13]. $\mathrm{P} 4 \mathrm{HB}$ has excellent thermal processability and can be fairly molded into various structures including fibers, films, tubes, and microspheres [10]. Tepha's TephaFLEX ${ }^{\circledR}$ is a P4HB biopolymer. Interestingly, the monofilament sutures fabricated using this $\mathrm{P} 4 \mathrm{HB}$ polymer was 35\% stronger than the synthetic polydioxanone suture and 19\% stronger than the polypropylene suture [14]. Therefore, $\mathrm{P} 4 \mathrm{HB}$ fibers have been used as the starting material for medical textile products like patches, grafts, surgical meshes, etc. [15]. Phasix ${ }^{\mathrm{TM}}$ mesh is a resorbable biosynthetic mesh prepared from P4HB [16]. Interestingly, it provided long-term mechanical strength during hernia repair surgery [17]. This might prevent further surgical complications and reduce the rate of hernia recurrence. It has also been observed that the P4HB-based surgical mesh reduced the chances of post-operative surgical site infections [18]. Thus, Phasix ${ }^{\mathrm{TM}}$ may become a treatment option for hernia repair. Furthermore, $\mathrm{P} 4 \mathrm{HB}$ are being successfully implemented in tissue engineering. Compared to polyglycolic acid (PGA) mesh, P4HB scaffolds showed prolonged degradation and also promoted tissue regeneration [19]. P4HB-based scaffold for trileaflet heart valves, vascular grafts, and artery augmentation, and $\mathrm{P} 4 \mathrm{HB}$ pelvic floor implants are already under consideration $[14,20]$. Moreover, degradation of $\mathrm{P} 4 \mathrm{HB}$ does not produce acidic by-products at wound sites and elicits mild inflammatory response during wound healing [21]. Due to its unique characteristics, $\mathrm{P} 4 \mathrm{HB}$ is recognized as a promising biodegradable and biocompatible biopolymer having immense potentials in biomedical engineering. However, high production cost is still an obvious challenge faced by PHA research. To commercialize the use of $\mathrm{P} 4 \mathrm{HB}$ and make it available for use in daily lives, it is important to curb down the production cost of $\mathrm{P} 4 \mathrm{HB}$.

\section{Microbial Synthesis of P4HB}

Several methods have been adopted to synthesize P4HB chemically. However, due to the involvement of various organometallic or metal complex catalysts such as Lewis acid, lanthanum amide, distannoxane complex, and titanium, chemical synthesis of $\mathrm{P} 4 \mathrm{HB}$ is not suitable for biomedical applications [10]. Thus, $\mathrm{P} 4 \mathrm{HB}$ synthesis through fermentation processes is the most desirable approach. Unlike PHB, a major drawback of microbial $\mathrm{P} 4 \mathrm{HB}$ production is that the naturally occurring P4HB-producing microbes are very limited. Most microbes can't produce the $\mathrm{P} 4 \mathrm{HB}$ homopolymer rather they tend to incorporate $3 \mathrm{HB}$ 
and/or $3 \mathrm{HV}$ monomers, resulting in the formation of $4 \mathrm{HB}$ copolymers or terpolymers. Both $4 \mathrm{HB}$ co- and terpolymers have novel and fascinating properties that are favoured over PHB and PHBV. Therefore, incorporating different proportions of $3 \mathrm{HB}, 3 \mathrm{HV}$, and $4 \mathrm{HB}$ monomers to yield tailor-made co- or terpolymer with desirable characteristics is an interesting research area. However, the fine balance between the monomeric units is critical. Sometimes, a high $3 \mathrm{HB}$ and $3 \mathrm{HV}$ content overshadows the benefits of $4 \mathrm{HB}$ content [22]. Therefore, optimizing the monomer composition is often challenging and requires extensive investigation. Taken together, a high $4 \mathrm{HB}$ content is always preferable and thus, continuous efforts are being made to synthesize PHA polymers having a high $4 \mathrm{HB}$ molar fraction. In addition, obtaining $4 \mathrm{HB}$ polymers at reduced cost is also a challenge faced by researchers in this field. In the following sections, different techniques like precursor supplementation, utilization of low-cost substrate, optimizing the feeding strategies and process parameters, and heterologous pathway construction to synthesize 4HB-containing polymers have been focused. Table 1 summarizes the production of $4 \mathrm{HB}$ polymer by different microorganisms.

\subsection{Natural 4 HB Polymer Producers and 4HB-CoA Supplying Pathway}

Hydrogenophaga pseudoflava is one of the few known microbes capable of producing $4 \mathrm{HB}$ polymer from structurally unrelated carbon sources. Using L-arabinose as the substrate, the strain synthesized the terpolymer $\mathrm{P}(3 \mathrm{HB}-\mathrm{co}-1 \mathrm{~mol} \% 3 \mathrm{HV}-\mathrm{co}-5 \mathrm{~mol} \% 4 \mathrm{HB})$ with a PHA content of $45.3 \mathrm{wt} \%$ (CDW) [23]. Thus, $H$. pseudoflava is a potentially important strain that should be further investigated to understand the metabolic pathway involved in $4 \mathrm{HB}$ polymer synthesis from unrelated carbon sources.

Synthesis of $4 \mathrm{HB}$ polymer requires generation of the $4 \mathrm{HB}-\mathrm{CoA}$ monomer. Using structurally unrelated carbon sources like glucose, $4 \mathrm{HB}-\mathrm{CoA}$ is synthesized from succinyl-CoA [13]. The synthesis of $4 \mathrm{HB}$ monomer from the succinate degradation pathway was characterized in Clostridium kluyveri [24]. In this pathway, succinate is converted to succinyl-CoA by succinyl-CoA: CoA transferase. The latter is then converted into succinate semialdehyde (SSA) by SSA dehydrogenase (Figure 1). The SSA is reduced to $4 \mathrm{HB}$ by $4 \mathrm{HB}$ dehydrogenase. 4HB-CoA: CoA transferase converts $4 \mathrm{HB}$ to $4 \mathrm{HB}-\mathrm{CoA}$. However, this strain is not able to synthesize $4 \mathrm{HB}$ polymer rather 4-hydroxybutyryl-CoA dehydratase/vinylacetyl-CoA $\Delta^{3}-\Delta^{2}$-isomerase converts $4 \mathrm{HB}-\mathrm{CoA}$ to crotonyl-CoA. For synthesis of $4 \mathrm{HB}$ polymer, under the action of PHA synthase, 4HB-CoA is incorporated into the polymer chain instead of channelizing it to other pathways. Most often, the genes involved in the 4HB-CoA generation pathway is absent or suppressed in microbes and thus, they are incapable of synthesizing $4 \mathrm{HB}$ polymer from unrelated carbon sources. This necessitates supplementation of structurally related $4 \mathrm{HB}$ precursors for the incorporation of $4 \mathrm{HB}$ monomers into polymer chain.

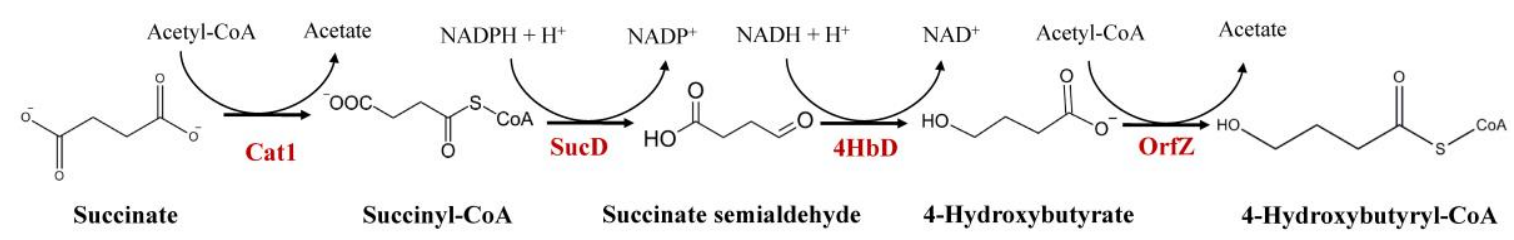

Figure 1. Naturally occurring 4HB-CoA supplying pathway in Clostridium kluyveri. Cat1, succinyl-CoA:CoA transferase; SucD, succinate semialdehyde dehydrogenase; $4 \mathrm{HbD}$, 4-hydroxybutyrate dehydrogenase; OrfZ, 4HB-CoA: CoA transferase. 
Table 1. 4HB polymers produced by different microorganisms.

\begin{tabular}{|c|c|c|c|c|c|}
\hline Microorganism & Genetic Modifications & Substrate & Polymer Type & Content (wt\% CDW) & Reference \\
\hline \multirow[b]{2}{*}{ Aneurinibacillus sp. H1 } & \multirow[t]{2}{*}{-} & Glycerol and 1,4-butanediol & $\mathrm{P}(3 \mathrm{HB}-\mathrm{co}-84 \mathrm{~mol} \%$ 4HB $)$ & - & [25] \\
\hline & & Glycerol, valerate, and 1,4-butanediol & $\begin{array}{c}\mathrm{P}(3 \mathrm{HB}-\mathrm{co}-33 \mathrm{~mol} \% \\
\text { 3HV-co-54 mol\% 4HB) }\end{array}$ & - & [25] \\
\hline Burkholderia sacchari & - & $\begin{array}{l}\text { Wheat straw hydrolysates and } \\
\gamma \text {-butyrolactone }\end{array}$ & $\mathrm{P}(3 \mathrm{HB}-\mathrm{co}-5 \mathrm{~mol} \%$ 4HB $)$ & 27 & [26] \\
\hline \multirow{6}{*}{ Cupriavidus necator } & \multirow{6}{*}{-} & $\begin{array}{c}\text { Citrate, ammonium sulphate, and } \\
\text { 4-hydroxybutyric acid }\end{array}$ & $\mathrm{P} 4 \mathrm{HB}$ & 2 & [27] \\
\hline & & $\begin{array}{l}\text { Propionic acid, ammonium sulphate, } \\
\text { and 4-hydroxybutyric acid }\end{array}$ & $\mathrm{P} 4 \mathrm{HB}$ & 34 & [28] \\
\hline & & Waste glycerol and $\gamma$-butyrolactone & $\mathrm{P}(3 \mathrm{HB}-\mathrm{co}-21.5 \mathrm{~mol} \%$ 4HB $)$ & 17.9 & [29] \\
\hline & & Soyabean oil and $\gamma$-butyrolactone & $\mathrm{P}(3 \mathrm{HB}-\mathrm{co}-10 \mathrm{~mol} \%$ 4HB $)$ & 80 & {$[30]$} \\
\hline & & $\begin{array}{l}\text { Waste glycerol, propionic acid, and } \\
\gamma \text {-butyrolactone }\end{array}$ & $\begin{array}{c}\mathrm{P}(3 \mathrm{HB}-c o-6 \mathrm{~mol} \% \\
3 \mathrm{HV}-c 0-43.6 \mathrm{~mol} \% \text { 4HB })\end{array}$ & 36.9 & [29] \\
\hline & & Fructose, Valerate, and 1,4-butanediol & $\begin{array}{c}\mathrm{P}(3 \mathrm{HB}-\mathrm{co}-16 \mathrm{~mol} \% \\
3 \mathrm{HV}-\mathrm{co}-51 \mathrm{~mol} \% \text { 4HB })\end{array}$ & 30 & {$[31]$} \\
\hline C. nectar (PHB leaky mutant) & - & 1,4-butanediol or 4-hydroxybutyric acid & $\mathrm{P} 4 \mathrm{HB}$ & 10 & [32] \\
\hline \multirow{3}{*}{ Cupriavidus malaysiensis } & \multirow{3}{*}{-} & 1,4-butanediol and $\gamma$-butyrolactone & $\mathrm{P}(3 \mathrm{HB}-\mathrm{co}-84 \mathrm{~mol} \%$ 4HB $)$ & 16 & [33] \\
\hline & & 1,4-butanediol and 1,6-hexanediol & $\mathrm{P}(3 \mathrm{HB}-\mathrm{co}-31 \sim 41 \mathrm{~mol} \%$ 4HB $)$ & 70 & [34] \\
\hline & & $\begin{array}{l}\text { Oleic acid, 1-pentanol, and } \\
\gamma \text {-butyrolactone }\end{array}$ & $\begin{array}{c}\mathrm{P}(3 \mathrm{HB}-\mathrm{co}-10 \mathrm{~mol} \% \\
\text { 3HV-co-9 mol\% 4HB) }\end{array}$ & 81 & {$[35]$} \\
\hline Cupriavidus sp. USMAHM13 & - & Glycerine pitch and 1,4-butanediol & $\mathrm{P}(3 \mathrm{HB}-\mathrm{co}-43 \mathrm{~mol} \%$ 4HB $)$ & 49 & {$[36]$} \\
\hline Comamonas acidovorans & - & 1,4-butanediol or 4-hydroxybutyric acid & $\mathrm{P} 4 \mathrm{HB}$ & 28 & [27] \\
\hline \multirow{2}{*}{ Haloferax mediterranei } & \multirow[t]{2}{*}{-} & Crude glycerol and $\gamma$-butyrolactone & $\begin{array}{l}\mathrm{P}(3 \mathrm{HB}-\mathrm{co}-10 \mathrm{~mol} \% \\
\text { 3HV-co-5 mol\% } 4 \mathrm{HB})\end{array}$ & 68.5 & {$[37]$} \\
\hline & & $\begin{array}{c}\text { Whey sugar, valerate and } \\
\gamma \text {-butyrolactone }\end{array}$ & $\begin{array}{l}\mathrm{P}(3 \mathrm{HB}-c o-21.8 \mathrm{~mol} \% \\
\text { 3HV-co-5.1 mol\% } 4 \mathrm{HB})\end{array}$ & 87.5 & [38] \\
\hline
\end{tabular}


Table 1. Cont.

\begin{tabular}{|c|c|c|c|c|c|}
\hline Microorganism & Genetic Modifications & Substrate & Polymer Type & Content (wt\% CDW) & Reference \\
\hline \multirow{2}{*}{ Hydrogenophaga pseudoflava } & \multirow[t]{2}{*}{-} & L-arabinose & $\begin{array}{c}\mathrm{P}(3 \mathrm{HB}-\mathrm{co}-1 \mathrm{~mol} \% 3 \mathrm{HV}-\mathrm{co}-5 \mathrm{~mol} \% \\
4 \mathrm{HB})\end{array}$ & 45.3 & {$[23]$} \\
\hline & & Cheese whey & $\begin{array}{c}\mathrm{P}(3 \mathrm{HB}-\mathrm{co}-18.4 \mathrm{~mol} \%- \\
4 \mathrm{HB}-\mathrm{co}-2.2 \mathrm{~mol} \%-3 \mathrm{HV})\end{array}$ & 2.9 & [39] \\
\hline \multirow{7}{*}{ Recombinant E. coli } & $\begin{array}{l}\text { phaCAB gene cluster from C. necator } \\
\text { and orfZ-sucD- } 4 h b D \text { from C. kluyveri } \\
\text { were co-expressed. }\end{array}$ & Glucose & $\mathrm{P}(3 \mathrm{HB}-\mathrm{co}-2.8 \mathrm{~mol} \%$ 4HB $)$ & 50 & {$[40]$} \\
\hline & $\begin{array}{l}\text { phaCAB gene cluster from } C \text {. necator, } \\
\text { orfZ-sucD-4hbD from C. kluyveri were } \\
\text { expressed; sad and gabD genes were } \\
\text { knocked out. }\end{array}$ & Glucose & $\mathrm{P}(3 \mathrm{HB}-c o-11.1 \mathrm{~mol} \%$ 4HB $)$ & 65.5 & [41] \\
\hline & $\begin{array}{l}\text { phaCAB gene cluster and phaP1 gene } \\
\text { from C. necator, orfZ-sucD-4hbD from } \\
\text { C. kluyveri were expressed; sad and } \\
\text { gabD genes were knocked out. }\end{array}$ & Glucose & $\mathrm{P} 4 \mathrm{HB}$ & 68 & [13] \\
\hline & $\begin{array}{l}\text { dhaT and aldD from P. putida KT2442, } \\
\text { orfZ from C. kluyveri, and phaC1 from } \\
\text { C. necator were expressed. }\end{array}$ & Glucose and 4-hydroxybutyric acid & P4HB & 83 & [42] \\
\hline & \multirow{2}{*}{$\begin{array}{l}\text { phaC from C. necator and orfZ from } \\
\text { C. kluyveri were expressed. }\end{array}$} & Xylose and 4-hydroxybutyric acid & $\mathrm{P} 4 \mathrm{HB}$ & 67 & [12] \\
\hline & & Glycerol and propionic acid & $\mathrm{P} 4 \mathrm{HB}$ & 80 & [43] \\
\hline & $\begin{array}{l}\text { orfZ from C. kluyveri, pcs' from } \\
\text { C. aurantiacus, dhaT and aldD from } \\
\text { P. putida KT2442, and phaC1 from } \\
\text { C. necator were expressed. }\end{array}$ & 1,3-propanediol and 1,4-butanediol & $\mathrm{P}(3 \mathrm{HP}-c o-30 \mathrm{~mol} \%$ 4HB) & 62.70 & {$[44]$} \\
\hline
\end{tabular}


Table 1. Cont.

\begin{tabular}{|c|c|c|c|c|c|}
\hline Microorganism & Genetic Modifications & Substrate & Polymer Type & Content (wt\% CDW) & Reference \\
\hline \multirow{5}{*}{$\begin{array}{l}\text { Recombinant } \\
\text { H. bluephagenesis }\end{array}$} & $\begin{array}{l}\text { orfZ gene from C. kluyveri was } \\
\text { chromosomally integrated. }\end{array}$ & Glucose and $\gamma$-butyrolactone & $\mathrm{P}(3 \mathrm{HB}-c o-16 \mathrm{~mol} \%$ 4HB $)$ & 61 & [45] \\
\hline & $\begin{array}{l}\text { Engineering the promoter driving } \\
\text { expression of chromosomally } \\
\text { integrated orfZ gene from C. kluyveri. }\end{array}$ & Glucose and $\gamma$-butyrolactone & $\mathrm{P}(3 \mathrm{HB}-c o-11 \mathrm{~mol} \%$ 4HB $)$ & 80 & [46] \\
\hline & $\begin{array}{l}\operatorname{ogd} A, \operatorname{sucD}, 4 h b d \text { and } \operatorname{orfZ} \text { were } \\
\text { introduced; gabD genes were } \\
\text { knocked out. }\end{array}$ & Glucose & $\mathrm{P}(3 \mathrm{HB}-c o-17 \mathrm{~mol} \%$ 4HB $)$ & 60.5 & [47] \\
\hline & \multirow{2}{*}{ orfZ chromosomally integrated. } & Glucose and $\gamma$-butyrolactone & $\mathrm{P}(3 \mathrm{HB}-\mathrm{co}-11.6 \mathrm{~mol} \%$ 4HB) & 73.8 & [48] \\
\hline & & Waste gluconate and $\gamma$-butyrolactone & $\mathrm{P}(3 \mathrm{HB}-\mathrm{co}-12.4 \mathrm{~mol} \%$ 4HB) & 70.6 & [48] \\
\hline Recombinant $C$. necator & $\begin{array}{c}\text { dhaT and aldD from P. putida KT2442 } \\
\text { were expressed. }\end{array}$ & $\begin{array}{l}\text { Fructose, 1,4-butanediol and } \\
\text { 4-hydroxybutyric acid }\end{array}$ & $\mathrm{P}(3 \mathrm{HB}-\mathrm{co}-13 \mathrm{~mol} \%$ 4HB $)$ & 49 & {$[42]$} \\
\hline Recombinant Bacillus megaterium & $\begin{array}{l}\text { sucD, } 4 h b D \text {, and orfZ from } C \text {. necator } \\
\text { under xylose inducible promoter was } \\
\text { introduced. }\end{array}$ & Glucose with xylose as inducer & $\mathrm{P}(3 \mathrm{HB}-\mathrm{co}-11 \mathrm{~mol} \%$ 4HB $)$ & $\sim 50$ & [49] \\
\hline
\end{tabular}




\subsection{Precursor Supplementation to Yield 4 HB Copolymers}

Precursor supplementation is the most important prerequisite for synthesis of $4 \mathrm{HB}$ polymers in many microbes. The commonly used 4HB precursors include $\gamma$-butyrolactone, 1,4-butanediol, and 1,6-hexanediol. These precursors are metabolized to form $4 \mathrm{HB}$, and then $4 \mathrm{HB}$ is converted to $4 \mathrm{HB}-\mathrm{CoA}$ by $4 \mathrm{HB}-\mathrm{CoA}$ : CoA transferase.

Studies on $4 \mathrm{HB}$ polymers dates back to late 1980s. For the first time, the synthesis of 4HB-containing PHA polymer was observed in Cupriavidus necator (also known as Ralstonia eutropha or Alcaligenes eutrophus) [51]. The strain produced PHB in the presence of carbon sources like fructose or butyric acid. However, co-feeding of $\gamma$-butyrolactone resulted in the synthesis of $\mathrm{P}(3 \mathrm{HB}-c o-4 \mathrm{HB})$ copolymer. $\gamma$-butyrolactone alone increased the $4 \mathrm{HB}$ content to $21 \mathrm{~mol} \%$ in the polymer. Co-feeding $\gamma$-butyrolactone with fructose decreased the $4 \mathrm{HB}$ content to $4 \mathrm{~mol} \%$ as fructose led to the formation of more $3 \mathrm{HB}-\mathrm{CoA}$ moieties. Strikingly, the mixture of $\gamma$-butyrolactone and butyric acid yielded a higher $4 \mathrm{HB}$ molar fraction $(24 \mathrm{~mol} \%)$, indicating that butyric acid also contributed to the formation of $4 \mathrm{HB}-\mathrm{CoA}$ monomer [51]. As the $4 \mathrm{HB}$ molar fraction increased in the copolymer, its crystallinity decreased and elongation to break increased. At $3 \mathrm{~mol} \%$ of $4 \mathrm{HB}$, crystallinity of the copolymer was $55 \%$ and elongation to break was $45 \%$. Interestingly at $16 \mathrm{~mol} \%$, the crystallinity decreased to $45 \%$ and the elongation to break increased to $444 \%$. Finally, due to the parameters' change, the physical properties of the polymer improved [51]. $\mathrm{P}(3 \mathrm{HB}-\mathrm{co}-4 \mathrm{HB})$ copolymer with a high $4 \mathrm{HB}$ molar fraction is more preferable for biomedical applications. Following this, several precursor feeding strategies have been explored for achieving a higher $4 \mathrm{HB}$ content in the PHA polymers. For instance, co-feeding citrate and ammonium sulphate with 4-hydroxybutyric acid led to the synthesis of P4HB homopolymer in C. necator. Unfortunately, the P4HB content was very low $(\approx 2 \mathrm{wt} \% \mathrm{CDW})$ [27]. Instead, the addition of $n$-alkanoic acid like propionic acid in the presence of ammonium sulphate and 4-hydroxybutyric acid increased the content of $\mathrm{P} 4 \mathrm{HB}$ homopolymer to $34 \mathrm{wt} \%(\mathrm{CDW})$ in the strain [28]. Comamonas acidovorans produced $\mathrm{P}(3 \mathrm{HB}-\mathrm{co}-4 \mathrm{HB})$ random copolymer from 4-hydroxybutyric acid and glucose or 3-hydroxybutyric acid [52]. Furthermore, it produced a terpolymer $\mathrm{P}(3 \mathrm{HB}-\mathrm{co}-3 \mathrm{HV}-\mathrm{co}-4 \mathrm{HB})$ from 1,4-butanediol and pentanol [53]. Interestingly, the strain produced $28 \mathrm{wt} \%$ (CDW) of P4HB homopolymer using 1,4-butanediol or 4-hydroxybutyric acid as the sole carbon source [27]. Alcaligenes latus is a natural PHB producer [54]. In the presence of sucrose and $\gamma$-butyrolactone, the strain produced $\mathrm{P}\left(3 \mathrm{HB}-\mathrm{co}^{-4 \mathrm{HB})}\right.$ [55]. The conversion efficacy of $\gamma$-butyrolactone to $4 \mathrm{HB}$ monomer was almost $60 \%$. Increasing the $\gamma$-butyrolactone concentration from 0.1 to $2.0 \mathrm{~g} / \mathrm{L}$, the $4 \mathrm{HB}$ content in polymers varied from 4 to $45 \mathrm{~mol} \%$. H. pseudoflava produces PHB from glucose [23]. Supplementation of $\gamma$-butyrolactone resulted in $4 \mathrm{HB}$ incorporation up to $66 \mathrm{~mol} \%$. Moreover, it also utilized $\varepsilon$-caprolactone as the $4 \mathrm{HB}$ precursor when co-fed with glucose to incorporate $20 \mathrm{~mol} \%$ of $4 \mathrm{HB}$ monomer. All these early studies established the importance of precursor supplementation for improving $4 \mathrm{HB}$ fraction in $4 \mathrm{HB}$-containing polymers.

Since 2000, more and more microbial strains have been explored to synthesize $4 \mathrm{HB}$ polymers using different composition of $4 \mathrm{HB}$ precursor mixture. Combination of 1,4-butanediol and $\gamma$-butyrolactone produced $\mathrm{P}(3 \mathrm{HB}-c o-4 \mathrm{HB})$ with a $4 \mathrm{HB}$ content as high as $84 \mathrm{~mol} \%$ in Cupriavidus malaysiensis [33]. C. malaysiensis produced $70 \mathrm{wt} \% \mathrm{P}(3 \mathrm{HB}-c o-4 \mathrm{HB})$ with $4 \mathrm{HB}$ fractions ranging from $31 \mathrm{~mol} \%$ to $41 \mathrm{~mol} \%$ by using a mixture of 1,4-butanediol and 1,6-hexanediol [34]. Co-feeding $\gamma$-butyrolactone and 1,6-hexanediol yielded a polymer with a higher $4 \mathrm{HB}$ molar fraction ( $55 \mathrm{~mol} \%$ ) in this strain [56]. Using carbon sources such as propionic acid or butyric acid with 4-hydroxybutyric acid in the presence of $\alpha$-amino acids, C. necator produced P(3HB-co-4HB) with 72-86 mol\% 4HB [57]. Propionic acid has a stimulating effect on $4 \mathrm{HB}$ polymer synthesis. The recombinant $E$. coli, engineered to synthesize $\mathrm{P} 4 \mathrm{HB}$, produced a higher content of $\mathrm{P} 4 \mathrm{HB}$ when propionic acid was supplied along with glycerol. The content increased from 30 to $80 \mathrm{wt} \%$ (CDW) when propionic acid concentration increased from 0 to $2 \mathrm{~g} / \mathrm{L}$ [43]. 


\subsection{Microbial Synthesis of $4 H B$ Terpolymer}

Studies on the synthesis of $4 \mathrm{HB}$ terpolymer is considerably limited. Till now, few microbes namely C. necator, C. malaysiensis, Aneurinibacillus sp. H1, H. pseudoflava, and haloarchaeon Haloferax mediterranei have been reported to synthesize terpolymer $\mathrm{P}(3 \mathrm{HB}-\mathrm{co}-3 \mathrm{HV}-\mathrm{co}-4 \mathrm{HB})$. Using 4-hydroxybutyric acid or $\gamma$-butyrolactone as $4 \mathrm{HB}$ precursor and propionic acid as $3 \mathrm{HV}$ precursor along with glucose, $C$. necator produced several types of the terpolymer $\mathrm{P}\left(3 \mathrm{HB}-\mathrm{co}-3 \mathrm{HV}-\mathrm{co}^{-4 \mathrm{HB})}\right.$ [58]. However, the terpolymer comprising approximately $10 \mathrm{~mol} \%$ of $3 \mathrm{HV}$ and $4 \mathrm{HB}$ each showed better mechanical properties than $\mathrm{P}(3 \mathrm{HB}-\mathrm{co}-30 \mathrm{~mol} \% 3 \mathrm{HV})$. Changing the carbon source type and ratio further changed the $4 \mathrm{HB}$ molar fraction in its terpolymer by using $C$. necator. For example, the supplementation of 4-hydroxybutyric acid to the medium containing fructose or butyric acid, and valerate resulted in the production of terpolymer containing $4 \mathrm{HB}$ up to $84 \mathrm{~mol} \%$ [31]. Moreover, the $4 \mathrm{HB}$ content further increased to $93 \mathrm{~mol} \%$ with increasing cultivation time. Replacing the $4 \mathrm{HB}$ precursor with 1,4-butanediol, however, reduced the $4 \mathrm{HB}$ content to almost $60 \mathrm{~mol} \%$ in this strain. A thermophilic gram-positive bacterium Aneurinibacillus sp. H1 accumulated PHB when cultured on glucose or glycerol. However, when supplemented with 1,4-butanediol, the strain produced $\mathrm{P}(3 \mathrm{HB}-\mathrm{co}-4 \mathrm{HB})$ with $4 \mathrm{HB}$ molar fraction up to $90 \mathrm{~mol} \%$. Moreover, by cofeeding valerate, it was able to synthesize terpolymer $\mathrm{P}(3 \mathrm{HB}-\mathrm{co}-33 \mathrm{~mol} \% 3 \mathrm{HV}-\mathrm{co}-54 \mathrm{~mol} \%$ 4HB) [25].

Ramachandran et al. (2011) conducted a detailed investigation to obtain a superior terpolymer in C. malaysiensis by varying the composition of $4 \mathrm{HB}$ and $3 \mathrm{HV}$ precursors [22]. A combination of 1,4-butanediol, 1-pentanol, and palmitic acid led to the synthesis of $\mathrm{P}(3 \mathrm{HB}-\mathrm{co}-4 \mathrm{~mol} \% 3 \mathrm{HV}-\mathrm{co}-33 \mathrm{~mol} \%$ 4HB) showing high Young's modulus (101 MPa) and elongation to break up to $937 \%$. Furthermore, P(3HB-co- $18 \mathrm{~mol} \% 3 \mathrm{HV}-\mathrm{co}-33 \mathrm{~mol} \% 4 \mathrm{HB})$ was synthesized by slightly increasing the concentration of 1-pentanol in the same substrate mixture and the polymer showed lower Young's modulus (34 $\mathrm{MPa}$ ) and reduced elongation to break (554\%). However, palmitic acid had a negative impact on the cell growth and thus reduced the PHA yield $(\approx 0.8 \mathrm{~g} / \mathrm{L})$. In contrast, oleic acid supported cell growth and improved PHA production to $3.3 \mathrm{~g} / \mathrm{L}$ by generating more acetyl-CoA via the $\beta$-oxidation pathway. Oleic acid favoured $3 \mathrm{HB}$ incorporation and hence reduced the $4 \mathrm{HB}$ content to almost $24 \mathrm{~mol} \%$ in the terpolymer [22,59]. Combining oleic acid and $\gamma$-butyrolactone with 1-pentanol, a high terpolymer content up to $81 \mathrm{wt} \%$ (CDW) was obtained [35]. Dissolved oxygen (DO)-stat fed-batch fermentation using the same substrate mixture was employed to improve the yield of terpolymer in C. malaysiensis [59]. Three different fed-batch fermentation produced $\mathrm{P}(3 \mathrm{HB}-\mathrm{co}-35 \mathrm{~mol} \% 3 \mathrm{HV}-\mathrm{co}-16 \mathrm{~mol} \% 4 \mathrm{HB})$, $\mathrm{P}(3 \mathrm{HB}-\mathrm{co}-16 \mathrm{~mol} \% 3 \mathrm{HV}-\mathrm{co}-22 \mathrm{~mol} \%$ 4HB), and $\mathrm{P}(3 \mathrm{HB}-\mathrm{co}-10 \mathrm{~mol} \% 3 \mathrm{HV}-\mathrm{co}-7 \mathrm{~mol} \% 4 \mathrm{HB})$ at a yield of 4.2, 8.2, and $16.7 \mathrm{~g} / \mathrm{L}$, respectively. Both $H$. pseudoflava and H. mediterranei synthesized $4 \mathrm{HB}$-containing terpolymer by utilizing low-cost substrates such as cheese whey and crude glycerol as discussed in the following section.

\subsection{Utilization of Low-Cost Substrate for 4HB Polymer Synthesis}

Synthesis of PHA is cost-intensive mainly due to the use of high-priced raw materials. Therefore, utilization of low-cost substrates for PHA synthesis has been a prominent research area. Usage of low-cost substrates such as food waste and agro-industrial wastes would not only contribute in curbing down the production cost, but would be a step towards waste valorization.

C. necator is able to synthesize $4 \mathrm{HB}$ polymer by using low-cost substrates including spent palm oil and waste glycerol. Using spent palm oil left after frying activities and 1,4-butanediol, the strain produced P(3HB-co- $15 \mathrm{~mol} \% 4 \mathrm{HB})$ [60]. Similarly, by using waste glycerol from biodiesel plant and $\gamma$-butyrolactone, $\mathrm{P}(3 \mathrm{HB}-\mathrm{co}-4 \mathrm{HB})$ with a $4 \mathrm{HB}$ incorporation up to $21.5 \mathrm{~mol} \%$ was achieved [29]. Moreover, co-feeding $\gamma$-butyrolactone with soybean oil, $C$. necator produced $\mathrm{P}(3 \mathrm{HB}-\mathrm{co}-4 \mathrm{HB})$ with $4 \mathrm{HB}$ molar fractions ranging from 6 to $10 \mathrm{~mol} \%$ [30]. Glycerine pitch is a routine waste generated from oleochemical industries. Cupriavidus sp. USMAHM13 produced $\mathrm{P}(3 \mathrm{HB}-c o-4 \mathrm{HB})$ with a $4 \mathrm{HB}$ molar fraction 
of $43 \mathrm{~mol} \%$ by using glycerine pitch and 1,4-butanediol [36]. Wheat straw hydrolysates serve as an excellent substrate for PHA production in Burkholderia sacchari. Co-feeding $\gamma$-butyrolactone with wheat straw hydrolysates, the strain accumulated $\mathrm{P}(3 \mathrm{HB}-\mathrm{co}-5 \mathrm{~mol} \%$ $4 \mathrm{HB}$ ) [26]. Using glucose and $\gamma$-butyrolactone, the recombinant $H$. bluephagenesis, capable of synthesizing $4 \mathrm{HB}$ polymer, produced $81.4 \mathrm{~g} / \mathrm{L} \mathrm{CDW}$ containing $73.8 \% \mathrm{P}(3 \mathrm{HB}-\mathrm{co}-11.6 \mathrm{~mol} \%$ $4 \mathrm{HB})$ [48]. When glucose was replaced with waste gluconate obtained from corn starch processing, the strain produced $68.1 \mathrm{~g} / \mathrm{L} \mathrm{CDW}$ containing $70.6 \% \mathrm{P}(3 \mathrm{HB}-\mathrm{co}-12.4 \mathrm{~mol} \% 4 \mathrm{HB})$. Not only the PHA yield was comparable to that obtained from glucose, but use of waste gluconate would reduce the raw material cost.

Synthesis of terpolymer using low-cost substrates have also been reported in few microbial species. In the presence of waste glycerol, $\gamma$-butyrolactone and propionic acid, C. necator produced $\mathrm{P}(3 \mathrm{HB}-\mathrm{co}-4 \mathrm{HB}-\mathrm{co}-3 \mathrm{HV})$ with $4 \mathrm{HB}$ molar fraction ranging from 24.8 to $43.6 \mathrm{~mol} \%$ and $3 \mathrm{HV} \%$ from 5.6 to $9.8 \mathrm{~mol} \%$ [29]. H. pseudoflava was able to synthesize the terpolymer $\mathrm{P}(3 \mathrm{HB}-\mathrm{co}-2.2 \mathrm{~mol} \% 3 \mathrm{HV}-\mathrm{co}-18.4 \mathrm{~mol} \% \mathrm{HB}$ ) using cheese whey as the sole carbon source [39]. In the Archaea domain, haloarchaeon H. mediterranei was capable of incorporating the $4 \mathrm{HB}$ monomers by using $4 \mathrm{HB}$ precursors. $H$. mediterranei is a natural producer of PHBV from structurally unrelated carbon sources such as glucose and chitin [61,62]. This strain is capable of producing $16.2 \mathrm{~g} / \mathrm{L}$ of $\mathrm{P}$ (3HB-co-10 mol\% 3HV) by utilizing crude glycerol from biodiesel production as the sole carbon source [37]. Supplying $\gamma$-butyrolactone with the crude glycerol led to the synthesis of $\mathrm{P}(3 \mathrm{HB}-\mathrm{co}-12 \mathrm{~mol} \%$ $3 \mathrm{HV}-\mathrm{co}-5 \mathrm{~mol} \% 4 \mathrm{HB}$ ) with a yield of $11.1 \mathrm{~g} / \mathrm{L}$. Similarly, in the presence of whey sugars as the sole carbon source, the strain synthesized $72.8 \mathrm{wt} \%$ of $\mathrm{P}(3 \mathrm{HB}-\mathrm{co}-6 \mathrm{~mol} \% 3 \mathrm{HV})$ [38]. The addition of valerate and $\gamma$-butyrolactone along with whey sugars resulted in the synthesis of $87.5 \mathrm{wt} \%$ of $\mathrm{P}(3 \mathrm{HB}-\mathrm{co}-21.8 \mathrm{~mol} \% 3 \mathrm{HV}-\mathrm{co}-5.1 \mathrm{~mol} \% 4 \mathrm{HB})$. Notably, PHA synthesis using $H$. mediterranei has some inherent advantages over bacteria. Fermentation of $H$. mediterranei does not involve stringent sterilization procedures because its high salinity requirement reduces chances of contamination [63]. Upon exposure to normal water, H. mediterranei cells undergo cell lysis which eases PHA recovery. Additionally, H. mediterranei has a tractable genetic system that provides the opportunity to improve $4 \mathrm{HB}$-containing terpolymer synthesis with the aid of metabolic engineering.

\subsection{Cultivation Parameters Affecting 4HB Polymer Synthesis}

Other than the type of carbon source, polymer synthesis is greatly affected by culture conditions and cultivation modes. Vigneswari et al., 2009 reported that cell concentration, phosphate ratio, and aeration in a culture influenced the $\mathrm{P}(3 \mathrm{HB}-\mathrm{co}-4 \mathrm{HB})$ synthesis as well as the $4 \mathrm{HB}$ molar fraction in C. malaysiensis [64]. With increasing cell concentration in the medium from 0.33 to $1.67 \mathrm{~g} / \mathrm{L}$, the $\mathrm{P}(3 \mathrm{HB}-\mathrm{co}-4 \mathrm{HB})$ yield increased from 0.58 to $1.25 \mathrm{~g} / \mathrm{L}$ and the $4 \mathrm{HB}$ molar fraction increased from 33 to $52 \mathrm{~mol} \%$ in this strain. When the phosphate ratio increased from 0 to 2.45 , the yield of $\mathrm{P}(3 \mathrm{HB}-\mathrm{co}-4 \mathrm{HB})$ increased from 0.21 to $2.49 \mathrm{~g} / \mathrm{L}$. Moreover, the $4 \mathrm{HB}$ molar fraction in polymers ranged between $29-54 \mathrm{~mol} \%$. Above a ratio of 2.45 , the polymer yield was slightly reduced. It was suggested that after a certain limit, phosphate concentration had a negative effect on polymer accumulation. Besides, culture aeration affected the $\mathrm{P}(3 \mathrm{HB}-\mathrm{co}-4 \mathrm{HB})$ yield and $4 \mathrm{HB}$ incorporation. Culture aeration decreased by increasing the culture volume at a fixed flask volume. With decreasing culture aeration, the polymer yield as well as $4 \mathrm{HB}$ molar fraction increased. Increasing the culture volume from 30 to $80 \mathrm{~mL}$ in a $250-\mathrm{mL}$ flask, the $4 \mathrm{HB}$ incorporation increased from 23 to $75 \mathrm{~mol} \%$. Thus, oxygen limitation facilitated $4 \mathrm{HB}$ incorporation. In fact, $\mathrm{P}(3 \mathrm{HB}-\mathrm{co}-4 \mathrm{HB})$ production in $30-\mathrm{L}$ reactor showed a higher $4 \mathrm{HB}$ incorporation $(40 \mathrm{~mol} \%)$ at lower agitation speed $(150 \mathrm{rpm})$ [65]. At higher agitation speeds (200 rpm and $250 \mathrm{rpm}$ ), $4 \mathrm{HB}$ incorporation was $\sim 28 \mathrm{~mol} \%$. In further studies, employing fed-batch cultivation strategies increased the $4 \mathrm{HB}$ molar fraction in an engineered C. malaysiensis [66]. Instead of feeding only carbon source, a pulse feed of carbon and nitrogen gave better performances. Twice pulse feed of carbon and nitrogen gave the best output, yielding $92 \mathrm{wt} \%$ $(\mathrm{CDW})$ of $\mathrm{P}(3 \mathrm{HB}-\mathrm{co}-99 \mathrm{~mol} \% 4 \mathrm{HB})$ with a PHA yield of $46 \mathrm{~g} / \mathrm{L}$. Moreover, maintaining 
a minimal level of nitrogen supplementation that is sufficient enough to support the cell growth required for polymer synthesis is recommended. Implementation of two-stage cultivation of $C$. malaysiensis is a promising approach to improve $4 \mathrm{HB}$ molar fraction in $\mathrm{P}(3 \mathrm{HB}-\mathrm{co}-3 \mathrm{HV}-\mathrm{co}-4 \mathrm{HB})$ and enhance the PHA production simultaneously. For instance, using $\gamma$-butyrolactone and 1-pentanol in two-stage cultivation, 4HB incorporation up to $14 \mathrm{~mol} \%$ and terpolymer concentration of $2.5 \mathrm{~g} / \mathrm{L}$ was achieved [67]. When the culture aeration was reduced, the $4 \mathrm{HB}$ molar fraction further increased to $24 \mathrm{~mol} \%$. In a further study, two-stage cultivation using glycerol with 1,4-butanediol in the first stage, and 1,4-butanediol with valerate in the second stage, $4.14 \mathrm{~g} / \mathrm{L}$ of $\mathrm{P}(3 \mathrm{HB}-\mathrm{co}-17.86 \mathrm{~mol} \%$ $3 \mathrm{HV}-\mathrm{co}-16.46 \mathrm{~mol} \% 4 \mathrm{HB}$ ) was obtained by using the same microbe [68].

The $4 \mathrm{HB}$ polymer production in C. necator was improved by employing DO-stat strategy for feeding the mixture of fructose and $\gamma$-butyrolactone [69]. In flask experiments, a $4 \mathrm{HB}$ molar fraction up to $27.4 \mathrm{~mol} \%$ was obtained by using fructose and $\gamma$-butyrolactone but the higher concentrations of both the substrates inhibited cell growth. Additionally, in the presence of a stimulator like propionate or acetate, a $4 \mathrm{HB}$ molar fraction of $53 \mathrm{~mol} \%$ was achieved but the PHA production $(0.27 \mathrm{~g} / \mathrm{L})$ was greatly reduced. However, the fed batch strategy improved polymer yield as PHA yield in the range of 14-24.4 g/L was obtained by varying the fructose to $\gamma$-butyrolactone ratio in the feeding solution. However, the $4 \mathrm{HB}$ molar fraction was less than the shake flask experiments and it ranged between 25.2 and $1.64 \mathrm{~mol} \%$. Similarly, the substrate ratio affected the $4 \mathrm{HB}$ molar fraction in $\mathrm{P}(3 \mathrm{HB}-\mathrm{co}-4 \mathrm{HB})$ in C. necator A-04 [70]. Using the mixture of $\gamma$-hydroxybutyric acid and butyric acid as carbon source and ammonium sulphate as nitrogen source, different monomer compositions were obtained by changing the $\mathrm{C} / \mathrm{N}$ ratio. At a high $\mathrm{C} / \mathrm{N}$ ratio, i.e., nitrogen deficient condition, $3 \mathrm{HB}$ incorporation was favoured whereas when the $\mathrm{C} / \mathrm{N}$ ratio was low (i.e., nitrogen sufficient condition), $4 \mathrm{HB}$ incorporation was preferred. $\mathrm{A}$ $\mathrm{C} / \mathrm{N}$ ratio of 4 resulted in a $4 \mathrm{HB}$ molar fraction of $70 \mathrm{~mol} \%$ in C. necator $\mathrm{A}-04$. The $4 \mathrm{HB}$ molar fraction in $\mathrm{P}(3 \mathrm{HB}-\mathrm{co}-4 \mathrm{HB})$ synthesized by Burkholderia sacchari was improved by finely tuning the fed batch strategy [71]. The $\gamma$-butyrolactone feeding was optimized to obtain the best outcome. A $\gamma$-butyrolactone pulse of $10 \mathrm{~g} / \mathrm{L}$ added at the initial polymer accumulation phase followed by its constant addition at a rate similar to its consumption ensured the maximal incorporation of $7.1 \mathrm{~mol} \% 4 \mathrm{HB}$. Taken together, synthesis of $4 \mathrm{HB}$ polymer containing desirable proportion of $4 \mathrm{HB}$ is directly related to the composition and concentration of the substrate mixture and the culture parameters.

\section{Metabolic Engineering to Synthesize 4HB Polymers}

Supplementation of $4 \mathrm{HB}$ precursor for synthesis of $4 \mathrm{HB}$-containing polymer incurs additional production cost. Moreover, some microbes including E. coli and Halomonas bluephagenesis TD01 are even incapable of utilizing $4 \mathrm{HB}$ precursors as carbon source. Thus, metabolic engineering is a better solution to enable $4 \mathrm{HB}$ polymer synthesis in such microbes. Pathway engineering that includes heterologous $4 \mathrm{HB}$ synthesis pathway de novo construction has successfully allowed $4 \mathrm{HB}$ polymer synthesis in several microbes.

\subsection{Construction of Recombinant E. coli to Synthesize 4HB Polymer}

Wild type E. coli is incapable of synthesizing PHA due to the lack of pha synthesis genes. However, PHB synthesis in E. coli has been made possible by pathway engineering. Similarly, recombinant $E$. coli was able to synthesize $4 \mathrm{HB}$ polymer when $4 \mathrm{HB}$ synthesis pathway was established in this strain (Figure 2). The entire PHA synthase gene (phaC) and 878 of 1179 -bp of the $5^{\prime}$ region of the $\beta$-ketothiolase gene (phaA) from $C$. necator plus the entire 4HB-CoA transferase gene (orf $Z$ ) from Clostridium kluyveri were heterologously expressed in E. coli [72]. Supplementing M9 mineral salt medium with 4-hydroxybutyric acid and glucose, the recombinant $E$. coli synthesized $\sim 80 \mathrm{wt} \%$ (CDW) P4HB homopolymer under oxygen-deficient condition. Even when replacing 4-hydroxybutyric acid with $\gamma$-butyrolactone, the E. coli strain synthesized $16.1 \mathrm{wt} \%$ (CDW) P4HB homopolymer. Another study by Valentin and Dennis (1997) reported that the phaCAB gene cluster (encoding 
PHA synthase, $\beta$-ketothiolase, and acetoacetyl-CoA reductase, respectively) from $C$. necator and orfZ-sucD-4hbD (encoding CoA transferase, succinate semialdehyde dehydrogenase, and 4-hydroxybutyrate dehydrogenase, respectively) from C. kluyveri were co-expressed in E. coli [40]. Using glucose as the sole carbon source, the engineered E. coli accumulated $50 \mathrm{wt} \%$ (CDW) $\mathrm{P}(3 \mathrm{HB}-\mathrm{co}-2.8 \mathrm{~mol} \% 4 \mathrm{HB})$. It was possible that due to the toxicity of succinate semialdehyde, formed from succinyl-CoA under the action of $s u c D$, succinate semialdehyde was degraded to succinate by its native succinate dehydrogenase in E. coli. This reduced the metabolic flux of glucose for 4HB-CoA generation. Thus, in another study by Li et al. (2010), sad and gabD genes were both knocked out to avert the degradation of succinate semialdehyde [41]. Interestingly, the resulting E. coli strain produced $65.5 \mathrm{wt} \%$ (CDW) $\mathrm{P}(3 \mathrm{HB}-\mathrm{co}-11.1 \mathrm{~mol} \%$ 4HB) using glucose as the sole carbon source. Furthermore, to promote the succinyl-CoA supplying pathway, TCA cycle intermediates such as 2-oxoglutarate, oxaloacetate, and citrate were supplemented in the media which increased the $4 \mathrm{HB} \mathrm{mol} \%$ to $\sim 22 \mathrm{~mol} \%, 16.3 \mathrm{~mol} \%$, and $20.3 \mathrm{~mol} \%$, respectively. Keeping the same genetic background, four phasin encoding genes (phaP1, phaP2, phaP3, and phaP4) from C. necator were individually expressed in the recombinant E. coli [13]. Interestingly, in the shake flasks containing LB medium supplemented with glucose, E. coli with no phaP gene synthesized only $12.13 \mathrm{wt} \%$ (CDW) P4HB whereas individual phaP expression increased the content to $\sim 22 \mathrm{wt} \%$ (CDW) with phaP2 or phaP4; $32.35 \mathrm{wt} \%$ (CDW) with phaP3, and $35.39 \mathrm{wt} \%$ (CDW) with phaP1. Furthermore, in fed-batch fermentation, $\sim 68 \mathrm{wt} \%$ (CDW) P4HB was produced by the recombinant $E$. coli with phaP1 expression. In another study, the 4-hydroxybutyric acid was first produced from 1, 4-butanediol by a recombinant Aeromonas hydrophila strain harbouring dhaT and aldD (encoding 1, 3-propanediol dehydrogenase and aldehyde dehydrogenase, respectively) from Pseudomonas putida KT2442 [42]. Then the 4HB-containing fermentation broth was used to synthesize P4HB in the recombinant E. coli. dhaT and aldD from P. putida KT2442, orfZ from C. kluyveri, and phaC1 from C. necator were simultaneously expressed in E. coli. The resultant recombinant strain produced $2 \mathrm{~g} / \mathrm{L}$ CDW containing $83 \mathrm{wt} \%$ of $\mathrm{P}(4 \mathrm{HB})$ when using glucose and the 4-hydroxybutyric acid as the carbon source. The presence of dhaT and aldD in E. coli helped in converting any residual 1,4-butanediol to $4 \mathrm{HB}$, thus, increasing the supply of $4 \mathrm{HB}$ for $\mathrm{P} 4 \mathrm{HB}$ synthesis.

Another novel copolymer of $4 \mathrm{HB}$ and 3-hydroxypropionic acid (3HP) has been synthesized in E. coli by using metabolic engineering strategy. P3HP is known for its high tensile strength and $\mathrm{P} 4 \mathrm{HB}$ for its elastic nature [73]. Thus, synthesis of $\mathrm{P}(3 \mathrm{HP}-\mathrm{co}-4 \mathrm{HB})$ possessing combined properties is a promising area in biomaterials. Synthesis of $3 \mathrm{HP}$ homopolymer from 1, 3-propandiol involved dhaT, aldD, pcs' (encoding ACS domain of propionyl-CoA synthase that functions like 3-hydroxypropanoic acid CoA ligase), and phaC genes [74]. Importantly, $4 \mathrm{HB}$ and $3 \mathrm{HP}$ are structurally similar and hence it was likely that the genes involved in the conversion of 3HP to $3 \mathrm{HP}-\mathrm{CoA}$ might be also functional in the conversion of 1, 4-butanediol to 4HB-CoA. Therefore, dhaT and aldD from P. putida KT2442, pcs' from Chloroflexus aurantiacus, phaC1 from C. necator were expressed in E. coli [74]. When supplemented with 1.3-propanediol and 1, 4-butanediol, the poly(3-hydroxypropionate-co-4-hydroxybutyrate) $\mathrm{P}(3 \mathrm{HP}-c o-4 \mathrm{HB})$ copolymer up to $42 \mathrm{wt} \%$ (CDW) was obtained. However, the $4 \mathrm{HB}$ molar fraction was less than $2 \mathrm{~mol} \%$. In another study, a different approach was adopted that led to a higher $4 \mathrm{HB}$ molar fraction (30 $\mathrm{mol} \%)$ in the copolymer [44]. The orfZ gene from C. kluyveri, pcs' from C. aurantiacus, dhaT and aldD from $P$. putida KT2442, and phaC1 from C. necator were expressed in E. coli. In the presence of 1.3-propanediol and 1, 4-butanediol, $62.70 \mathrm{wt} \%$ (CDW) P (70 mol\% 3HP-co-30 mol\% $4 \mathrm{HB})$ was synthesized. The thermal properties of the $4 \mathrm{HB}$ copolymers were improved as the melting point was reduced with the incorporation of $4 \mathrm{HB}$ monomers compared to P3HP. Moreover, the elongation at break of the $4 \mathrm{HB}$ copolymer also increased compared to P3HP. However, the tensile strength and Young's modulus of the copolymer did not show improvements except that the copolymer containing $12 \mathrm{~mol} \% 4 \mathrm{HB}$ showed a higher tensile strength compared to P3HP. Interestingly, the copolymer containing $67 \mathrm{~mol} \%$ of 
4HB was completely transparent unlike $\mathrm{P} 3 \mathrm{HP}$, thus, necessitating further investigation on the synthesis of $\mathrm{P}(3 \mathrm{HP}-c o-4 \mathrm{HB})$.

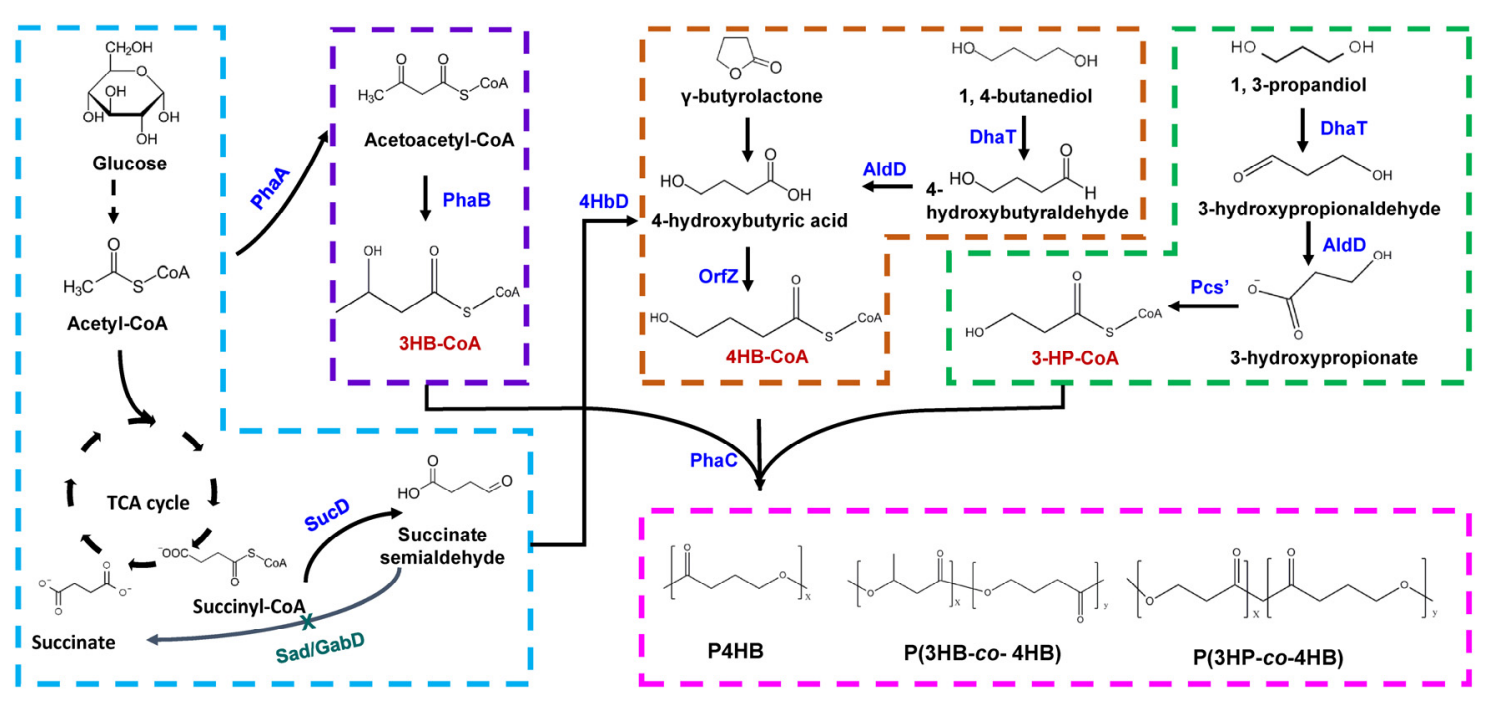

Figure 2. Different metabolic engineering strategies to synthesize 4HB-containing PHA in Escherichia coli. When using glucose as the sole substrate, it is converted to succinyl-CoA via glycolytic pathway and TCA cycle. Succinyl-CoA is converted to succinate semialdehyde by SucD. Deletion of native sad/gabD genes prevents the conversion of succinate semialdehyde to succinate. Then, succinate semialdehyde is converted to 4 -hydroxybutyric acid by $4 \mathrm{HbD}$. When using $\gamma$-butyrolactone or 1, 4-butanediol as $4 \mathrm{HB}$ precursor, they are converted to 4-hydroxybutyric acid. 4-hydroxybutyric acid is then converted to $4 \mathrm{HB}-\mathrm{CoA}$ by OrfZ. 3HB-CoA is derived from acetyl-CoA under the action of PhaA and PhaB. Finally, $\mathrm{P} 4 \mathrm{HB}$ or $\mathrm{P}(3 \mathrm{HB}-c o-4 \mathrm{HB})$ is synthesized under the action of PhaC. For the synthesis of $\mathrm{P}(3 \mathrm{HP}-c o-4 \mathrm{HB}), 3 \mathrm{HP}-\mathrm{CoA}$ is derived from 1,3-propandiol. Under the action of PhaC, 3HP-CoA and 4HB-CoA is polymerized to form P (3HP-co-4HB). SucD, succinate semialdehyde dehydrogenase; $\mathrm{Sad}$ and $\mathrm{GabD}$, succinate semialdehyde dehydrogenase; PhaA, $\beta$-ketothiolase; PhaB, acetoacetyl-CoA reductase; PhaC, PHA synthase; 4hbD, 4-hydroxybutyrate dehydrogenase; OrfZ, CoA transferase; DhaT, 1,3-propanediol dehydrogenase; AldD, aldehyde dehydrogenase; Pcs', ACS domain of propionyl-CoA synthase.

\subsection{Engineering H. bluephagenesis TD01 for Synthesis of 4HB Polymer}

H. bluephagenesis TD01 is a halophilic bacterium showing a high capability in PHA industrial production, however, it is unable to synthesize 4HB-containing PHA even in the presence of $4 \mathrm{HB}$ precursor. The $4 \mathrm{HB}-\mathrm{CoA}$ transferase encoding gene is absent in this microbe. Hence, the orfZ gene from C. kluyveri was expressed in H. bluephagenesis TD01(Figure 3) [45]. Unlike E. coli, H. bluephagenesis TD01 possesses its own pha synthesis genes. The T7-like system was used to tune the orfZ expression strength. In the presence of glucose and $\gamma$-butyrolactone, the recombinant strain incorporated $11.2 \mathrm{~mol} \%$ of $4 \mathrm{HB}$ in $\mathrm{P}(3 \mathrm{HB}-\mathrm{co}-4 \mathrm{HB})$. To ensure better genetic stability, orf $\mathrm{Z}$ was then chromosomally integrated in the genome of $H$. bluephagenesis TD01. In a 7-L fermentor, $72 \mathrm{~g} / \mathrm{L}$ CDW containing $63 \% \mathrm{P}(3 \mathrm{HB}-\mathrm{co}-12.3 \mathrm{~mol} \% \mathrm{HHB})$ was produced in $48 \mathrm{~h}$. To further scale-up the process, the fermentation was carried out in 1000-L fermentor in an open unsterile condition. After $48 \mathrm{~h}$ of fermentation, $82.6 \mathrm{~g} / \mathrm{L} \mathrm{CDW}$ containing $61 \% \mathrm{P}(3 \mathrm{HB}-\mathrm{co}-16 \mathrm{~mol} \% \mathrm{HHB})$ was achieved [45]. Furthermore, by engineering the promoter driving the expression of orf $\mathrm{Z}$ gene, $100 \mathrm{~g} / \mathrm{L}$ CDW containing $80 \% \mathrm{P}(3 \mathrm{HB}-\mathrm{co}-11 \mathrm{~mol} \% \mathrm{HHB})$ was obtained in unsterile fed-batch fermentation [46]. Finally, to synthesize $\mathrm{P}(3 \mathrm{HB}-\mathrm{co}-4 \mathrm{HB})$ using only glucose, the orfZ gene and $\operatorname{ogdA}$ encoding 2-oxo-glutarate dehydrogenase, sucD and $4 \mathrm{hbd}$ genes were introduced in H. bluephagenesis TD01 (Figure 3) [47]. This would direct succinate semialdehyde derived from 2-oxoglutarate and succinyl-CoA in the TCA cycle towards 4HB-CoA generation. Simultaneously, gabD genes were deleted to avoid the conversion of succinate semialdehyde to succinate [47]. The resulting strain was able to produce $26.3 \mathrm{~g} / \mathrm{L} \mathrm{CDW}$ containing $60.5 \% \mathrm{P}(3 \mathrm{HB}-\mathrm{co}-17 \mathrm{~mol} \% 4 \mathrm{HB})$ in 7 -L bioreactor after $60 \mathrm{~h}$ of fed-batch fermentation under 
unsterile conditions. Moreover, by controlling the concentration of residual glucose in the culture, the $4 \mathrm{HB}$ fraction varied from 13.4 to $24.9 \mathrm{~mol} \%$. Thus, H. bluephagenesis TD01 holds high promise as an efficient industrial producer of $\mathrm{P}(3 \mathrm{HB}-\mathrm{co}-4 \mathrm{HB})$ polymer.



Figure 3. Metabolic engineering of Halomonas bluephagenesis TD01 to synthesize P (3HB-co-4HB). When using $\gamma$-butyrolactone as $4 \mathrm{HB}$ precursor, 4-hydroxybutyric acid is converted to $4 \mathrm{HB}-\mathrm{CoA}$ by OrfZ. 3HB-CoA is derived from acetyl-CoA under the action of PhaA and PhaB. When using glucose as the sole substrate, it is converted to succinyl-CoA via glycolytic pathway and TCA cycle. OgdA catalyzes the conversion of 2-oxoglutarate to succinyl-CoA which is further converted to succinate semialdehyde by SucD. Deletion of $g a b D$ genes prevented the conversion of succinate semialdehyde to succinate. Next, succinate semialdehyde is converted to 4-hydroxybutyric acid by $4 \mathrm{HbD}$. Under the action of $\mathrm{PhaC}, 3 \mathrm{HB}-\mathrm{CoA}$ and $4 \mathrm{HB}-\mathrm{CoA}$ are polymerized to form $\mathrm{P}(3 \mathrm{HB}-\mathrm{co}-4 \mathrm{HB})$. OgdA, 2-oxoglutarate dehydrogenase; SucD, succinate semialdehyde dehydrogenase; GabD, succinate semialdehyde dehydrogenase; $4 \mathrm{HbD}$, 4-hydroxybutyrate dehydrogenase; OrfZ, CoA transferase; PhaA, $\beta$-ketothiolase; PhaB, acetoacetyl-CoA reductase; PhaC, PHA synthase.

\subsection{Synthesis of $4 H B$ Polymer in Other Microbes}

A PHB-leaky transposon-induced mutant strain of C. nectar JMP222 accumulated less PHB compared to the wild type strain. Notably, all the three PHB biosynthetic enzymes, PhaA, PhaB, and PhaC were synthesized at normal levels [75]. It was speculated that the mobilization system of the PHB-leaky mutant was affected as its PHB content dropped rapidly once the carbon source was exhausted compared to the wild-type [75]. Interestingly, the PHB-leaky mutant strain synthesized $~ 10 \mathrm{wt} \%$ (CDW) P4HB by using 1,4-butanediol or 4-hydroxybutyric acid [32]. Interestingly, overexpressing the phaC gene from wild-type $C$. nectar in the mutant strain increased the P4HB content to $\sim 30 \mathrm{wt} \%$ (CDW). Co-expression of dhaT and aldD from P. putida KT2442 in C. nectar led to the synthesis of $6.1 \mathrm{~g} / \mathrm{L} \mathrm{CDW}$ containing $49 \mathrm{wt} \% \mathrm{P}(3 \mathrm{HB}-\mathrm{co}-13 \mathrm{~mol} \% 4 \mathrm{HB})$ in LB medium with fructose, 1,4-butanediol and 4-hydroxybutyric acid (produced by recombinant $A$. hydrophila) [42]. C. malaysiensis was able to synthesize $\mathrm{P}(3 \mathrm{HB}-\mathrm{co}-4 \mathrm{HB})$ with a $4 \mathrm{HB}$ molar fraction up to $75 \%$ using different $4 \mathrm{HB}$ precursors [76]. The $4 \mathrm{HB}$ molar fraction was further enhanced by genetic engineering. The $p h a C$ gene of $C$. malaysiensis USMAA1020 and C. malaysiensis 
USMAA2-4 were cloned and introduced into the wild type $C$. malaysiensis USMAA1020, respectively. This led to the $\mathrm{P}(3 \mathrm{HB}-\mathrm{co}-4 \mathrm{HB})$ synthesis with $93 \mathrm{~mol} \%$ of $4 \mathrm{HB}$ [76]. Burkholderia sp. is capable of accumulating PHA and its pha gene cluster consists of phaC, phaA, phaB, and phaR (encoding the PHA synthesis regulator). However, it does not have the ability to produce $4 \mathrm{HB}$ polymer. Nevertheless, when the $\mathrm{phaC}$ gene of Burkholderia sp. strain JCM 15050 was expressed in the PHA-negative $C$. necator strain $\mathrm{PHB}^{-} 4$, the latter accumulated $\mathrm{P}(3 \mathrm{HB}-\mathrm{co}-4 \mathrm{HB})$ with a $4 \mathrm{HB}$ molar fraction up to $87 \mathrm{~mol} \%$ using sodium 4-hydroxybutyrate [77]. Similarly, the heterologous expression of phaC from Burkholderia contaminans Kad1 in C. necator strain $\mathrm{PHB}^{-} 4$ allowed the latter to produce $\mathrm{P}(3 \mathrm{HB}-c o-4 \mathrm{HB})$ with a $4 \mathrm{HB}$ content up to $51 \mathrm{~mol} \%$ from sodium 4-hydroxybutyrate [78]. The cell wall of Bacillus species lacks lipopolysaccharides and a novel self-destructive cell lysis system was established which eases the PHA recovery process $[49,79]$. This makes Bacillus species a promising host for PHA synthesis. Unfortunately, Bacillus species is incompetent of producing $4 \mathrm{HB}$ polymer from related carbon sources [49]. However, there are some reports showing the natural synthesis of $4 \mathrm{HB}$ polymer in Bacillus cereus from unrelated carbon sources like fructose and gluconate [80]. With the aid of metabolic engineering, $4 \mathrm{HB}$ polymer synthesis has been realized in Bacillus megaterium [49]. To synthesize P (3HB-co-4HB), sucD, 4hbD, and orf $Z$ from $C$. kluyveri under the control of xylose-inducible promoter were expressed in B. megaterium. Interestingly, a $4 \mathrm{HB}$ of $10 \mathrm{~mol} \%$ was incorporated using glucose as the carbon source and xylose as the inducer. Hu et al. (2011) synthesized a PHA block copolymer consisting of PHB and P4HB blocks [81]. The phaC and orfZ from C. nectar was expressed in a $\beta$-oxidation weakened strain of $P$. putida. The resulting recombinant strain synthesized di-block copolymer in which one block was PHB and another was P4HB [81]. Cyanobacteria Synechococcus sp. PCC 7002 was genetically engineered to synthesize $\mathrm{P}\left(3 \mathrm{HB}-\mathrm{co}^{-} \mathrm{HHB}\right)$. To realize this, the non-PHA producing wild-type strain was first engineered to synthesize $\mathrm{PHB}$ and then the biosynthetic pathway for synthesis of $\mathrm{P}(3 \mathrm{HB}-\mathrm{co}-4 \mathrm{HB})$ was constructed in the PHB-producing strain [50]. Precisely, the phaABEC operon encoding acetyl-CoA acetyltransferase, acetoacetyl-CoA reducatese, class III PHA synthase (PhaE subunit and PhaC subunit), respectively, from Chlorogloeopsis fritschii was expressed in Synechococcus sp. PCC 7002. The resulting strain synthesized PHB. Further deletion of the $c \mathrm{cmR}$ gene encoding LysR-type transcription factor improved the acetyl-CoA pool that improved PHB synthesis. Finally, in this engineered strain, a 4HB synthesis pathway containing the 4-hydroxybutyrate dehydrogenase ( $g b d 1)$ and 4-hydroxybutyryl-CoA transferase (cat2) genes from Porphyromonas gingivalis and the native 2-oxoglutarate decarboxylase $(\operatorname{og} d A)$ gene was introduced. The resulting strain accumulated $4.5 \mathrm{wt} \%$ (CDW) containing P(3HB-co-12 mol\% 4HB).

\section{Conclusion and Future Perspectives}

P4HB or 4HB-containing PHA is a promising biomaterial in the biomedical field. However, its synthesis is a constraint as most microbes lack $4 \mathrm{HB}$ synthesis pathways. To commercialize the use of $\mathrm{P} 4 \mathrm{HB}$ or its copolymers, it is necessary to accelerate its production. Researchers have adopted different strategies such as supplementing structurally related $4 \mathrm{HB}$ precursors such as 1,4-butanediol, $\gamma$-butyrolactone, and 4-hydroxybutyric acid. In addition, several research groups have employed low-cost substrates for producing $4 \mathrm{HB}$ polymer which would contribute to reducing the production cost of the polymer. Optimizing the cultivation parameters like feeding strategy, mode of fermentation, $\mathrm{C} / \mathrm{N}$ ratio, and aeration status also help in improving the production of $4 \mathrm{HB}$ polymer. However, some microbes are incapable of utilizing $4 \mathrm{HB}$ precursors, thus, necessitating application of metabolic engineering. The $4 \mathrm{HB}$ synthesis pathways have been established in several microbes. Among them, $\mathrm{H}$. bluephagenesis TD01 is an industrially proficient producer as the engineered $H$. bluephagenesis strain can produce $4 \mathrm{HB}$ polymer from glucose and can also be cultivated in open, and unsterile large scale fermentor. Nevertheless, more and more microbes synthesizing $4 \mathrm{HB}$ polymer should be explored to commercialize the use of $4 \mathrm{HB}$ polymer in global market. Moreover, since the use of $4 \mathrm{HB}$ precursor incur 
additional production cost, utilization of cheap sugars to synthesize $4 \mathrm{HB}$ polymer should be prioritized.

Author Contributions: Writing-original draft preparation, R.M. and J.H.; writing-review and editing, R.M., J.H. and H.X.; supervision, J.H. and H.X. All authors have read and agreed to the published version of the manuscript.

Funding: This work was financially supported by grants from Funding from National Key R\&D Program of China (No. 2020YFA0906800), the National Natural Science Foundation of China (No. 31970031), and CAS-TWAS President's Fellowship (to RM).

Institutional Review Board Statement: Not applicable.

Informed Consent Statement: Not applicable.

Data Availability Statement: Not applicable.

Acknowledgments: The authors would also like to thank Guomin Ai for help in compound structure preparation.

Conflicts of Interest: The authors declare no conflict of interests.

\section{References}

1. Accinelli, C.; Saccà, M.L.; Mencarelli, M.; Vicari, A. Deterioration of bioplastic carrier bags in the environment and assessment of a new recycling alternative. Chemosphere 2012, 89, 136-143. [CrossRef]

2. Kumar, V.; Kumar, S.; Singh, D. Microbial polyhydroxyalkanoates from extreme niches: Bioprospection status, opportunities and challenges. Int. J. Biol. Macromol. 2020, 147, 1255-1267. [CrossRef]

3. Reddy, C.S.K.; Ghai, R.; Kalia, V. Polyhydroxyalkanoates: An overview. Bioresour. Technol. 2003, 87, 137-146. [CrossRef]

4. Chen, G.Q.; Wu, Q. The application of polyhydroxyalkanoates as tissue engineering materials. Biomaterials 2005, 26, 6565-6578. [CrossRef] [PubMed]

5. Steinbüchel, A.; Lütke-Eversloh, T. Metabolic engineering and pathway construction for biotechnological production of relevant polyhydroxyalkanoates in microorganisms. Biochem. Eng. J. 2003, 16, 81-96. [CrossRef]

6. Huang, P.; Okoshi, T.; Mizuno, S.; Hiroe, A.; Tsuge, T. Gas chromatography-mass spectrometry-based monomer composition analysis of medium-chain-length polyhydroxyalkanoates biosynthesized by Pseudomonas spp. Biosci. Biotechnol. Biochem. 2018, 82, 1615-1623. [CrossRef]

7. McAdam, B.; Brennan Fournet, M.; McDonald, P.; Mojicevic, M. Production of polyhydroxybutyrate (PHB) and factors impacting its chemical and mechanical characteristics. Polymers 2020, 12, 2908. [CrossRef] [PubMed]

8. Barbosa, J.L.; Perin, G.B.; Felisberti, M.I. Plasticization of Poly (3-hydroxybutyrate-co-3-hydroxyvalerate) with an Oligomeric Polyester: Miscibility and Effect of the Microstructure and Plasticizer Distribution on Thermal and Mechanical Properties. ACS Omega 2021, 6, 3278-3290. [CrossRef]

9. Han, J.; Wu, L.P.; Liu, X.B.; Hou, J.; Zhao, L.L.; Chen, J.Y.; Zhao, D.H.; Xiang, H. Biodegradation and biocompatibility of haloarchaea-produced poly (3-hydroxybutyrate-co-3-hydroxyvalerate) copolymers. Biomaterials 2017, 139, 172-186. [CrossRef] [PubMed]

10. Utsunomia, C.; Ren, Q.; Zinn, M. Poly (4-hydroxybutyrate): Current state and perspectives. Front. Bioeng. Biotechnol. 2020, 8, 257. [CrossRef]

11. Martin, D.P.; Badhwar, A.; Shah, D.V.; Rizk, S.; Eldridge, S.N.; Gagne, D.H.; Ganatra, A.; Darois, R.E.; Williams, S.F.; Tai, H.C.; et al. Characterization of poly-4-hydroxybutyrate mesh for hernia repair applications. J. Surg. Res. 2013, 184, 766-773. [CrossRef]

12. Le Meur, S.; Zinn, M.; Egli, T.; Thöny-Meyer, L.; Ren, Q. Poly (4-hydroxybutyrate) (P4HB) production in recombinant Escherichia coli: P4HB synthesis is uncoupled with cell growth. Microb. Cell Fact. 2013, 12, 1-11. [CrossRef]

13. Zhou, X.Y.; Yuan, X.X.; Shi, Z.Y.; Meng, D.C.; Jiang, W.J.; Wu, L.P.; Chen, J.C.; Chen, G.Q. Hyperproduction of poly (4-hydroxybutyrate) from glucose by recombinant Escherichia coli. Microb. Cell Fact. 2012, 11, 1-8. [CrossRef]

14. Martin, D.P.; Williams, S.F. Medical applications of poly-4-hydroxybutyrate: A strong flexible absorbable biomaterial. Biochem. Eng. J. 2003, 16, 97-105. [CrossRef]

15. Wu, Q.; Wang, Y.; Chen, G.Q. Medical application of microbial biopolyesters polyhydroxyalkanoates. Artif. Cells Blood Substit. Biotechnol. 2009, 37, 1-12. [CrossRef] [PubMed]

16. Van Rooijen, M.M.J.; Jairam, A.P.; Tollens, T.; Jørgensen, L.N.; de Vries Reilingh, T.S.; Piessen, G.; Köckerling, F.; Miserez, M.; Windsor, A.C.J.; Berrevoet, F.; et al. A post-market, prospective, multi-center, single-arm clinical investigation of Phasix ${ }^{\mathrm{TM}}$ mesh for VHWG grade 3 midline incisional hernia repair: A research protocol. BMC Surgery 2018, 18, 1-9. [CrossRef] [PubMed]

17. Scott, J.R.; Deeken, C.R.; Martindale, R.G.; Rosen, M.J. Evaluation of a fully absorbable poly-4-hydroxybutyrate/absorbable barrier composite mesh in a porcine model of ventral hernia repair. Surg. Endosc. 2016, 30, 3691-3701. [CrossRef]

18. Molina, C.P.; Hussey, G.S.; Liu, A.; Eriksson, J.; D’Angelo, W.A.; Badylak, S.F. Role of 4-hydroxybutyrate in increased resistance to surgical site infections associated with surgical meshes. Biomaterials 2021, 267, 120493. [CrossRef] [PubMed] 
19. Dvorin, E.L.; Wylie-Sears, J.; Kaushal, S.; Martin, D.P.; Bischoff, J. Quantitative Evaluation of Endothelial Progenitors and Cardiac Valve Endothelial Cells: Proliferation and Differentiation on Poly-glycolic acid/Poly-4-hydroxybutyrate Scaffold in Response to Vascular Endothelial Growth Factor and Transforming Growth Factor $\beta_{1}$. Tissue Eng. 2003, 9, 487-493.

20. Verhorstert, K.W.; Guler, Z.; de Boer, L.; Riool, M.; Roovers, J.P.W.; Zaat, S.A. In Vitro Bacterial Adhesion and Biofilm Formation on Fully Absorbable Poly-4-hydroxybutyrate and Nonabsorbable Polypropylene Pelvic Floor Implants. ACS Appl. Mater. Interfaces 2020, 12, 53646-53653. [CrossRef]

21. Deeken, C.R.; Matthews, B.D. Characterization of the mechanical strength, resorption properties, and histologic characteristics of a fully absorbable material (Poly-4-hydroxybutyrate-PHASIX Mesh) in a porcine model of hernia repair. Int. Sch. Res. Not. 2013, 2013, 1-12. [CrossRef] [PubMed]

22. Ramachandran, H.; Iqbal, N.M.; Sipaut, C.S.; Abdullah, A.A.A. Biosynthesis and characterization of poly (3-hydroxybutyrate-co-3-hydroxyvalerate-co-4-hydroxybutyrate) terpolymer with various monomer compositions by Cupriavidus sp. USMAA2-4. Appl. Biochem. Biotechnol. 2011, 164, 867-877. [CrossRef] [PubMed]

23. Choi, M.H.; Song, J.J.; Yoon, S.C. Biosynthesis of copolyesters by Hydrogenophaga pseudoflava from various lactones. Can. J. Microbiol. 1995, 41, 60-67. [CrossRef]

24. Söhling, B.; Gottschalk, G. Molecular analysis of the anaerobic succinate degradation pathway in Clostridium kluyveri. J. Bacteriol. 1996, 178, 871-880. [CrossRef]

25. Sedlacek, P.; Pernicova, I.; Novackova, I.; Kourilova, X.; Kalina, M.; Kovalcik, A.; Koller, M.; Nebesarova, J.; Krzyzanek, V.; Hrubanova, K.; et al. Introducing the newly isolated bacterium Aneurinibacillus sp. H1 as an auspicious thermophilic producer of various polyhydroxyalkanoates (PHA) copolymers-2. Material study on the produced copolymers. Polymers 2020, $12,1298$. [CrossRef]

26. Cesário, M.T.; Raposo, R.S.; de Almeida, M.C.M.; Van Keulen, F.; Ferreira, B.S.; Telo, J.P.; da Fonseca, M.M.R. Production of poly (3-hydroxybutyrate-co-4-hydroxybutyrate) by Burkholderia sacchari using wheat straw hydrolysates and gamma-butyrolactone. Int. J. Biol. Macromol. 2014, 71, 59-67. [CrossRef] [PubMed]

27. Saito, Y.; Nakamura, S.; Hiramitsu, M.; Doi, Y. Microbial synthesis and properties of poly (3-hydroxybutyrate-co-4-hydroxybutyrate). Polym. Int. 1996, 39, 169-174. [CrossRef]

28. Kimura, H.; Ohura, T.; Takeishi, M.; Nakamura, S.; Doi, Y. Effective microbial production of poly (4-hydroxybutyrate) homopolymer by Ralstonia eutropha H16. Polym. Int. 1999, 48, 1073-1079. [CrossRef]

29. Cavalheiro, J.M.; Raposo, R.S.; de Almeida, M.C.M.; Cesário, M.T.; Sevrin, C.; Grandfils, C.; Da Fonseca, M.M.R. Effect of cultivation parameters on the production of poly (3-hydroxybutyrate-co-4-hydroxybutyrate) and poly (3-hydroxybutyrate-4-hydroxybutyrate-3-hydroxyvalerate) by Cupriavidus necator using waste glycerol. Bioresour. Technol. 2012, 111, 391-397. [CrossRef]

30. Park, D.H.; Kim, B.S. Production of poly (3-hydroxybutyrate) and poly (3-hydroxybutyrate-co-4-hydroxybutyrate) by Ralstonia eutropha from soybean oil. New Biotechnol. 2011, 28, 719-724. [CrossRef]

31. Chanprateep, S.; Kulpreecha, S. Production and characterization of biodegradable terpolymer poly (3-hydroxybutyrate-co-3-hydroxyvalerate-co-4-hydroxybutyrate) by Alcaligenes sp. A-04. J. Biosci. Bioeng. 2006, 101, 51-56. [CrossRef]

32. Steinbüchel, A.; Valentin, H.E.; Schönebaum, A. Application of recombinant gene technology for production of polyhydroxyalkanoic acids: Biosynthesis of poly (4-hydroxybutyric acid) homopolyester. J. Environ. Polym. Degrad. 1994, 2, 67-74. [CrossRef]

33. Vigneswari, S.; Nik, L.A.; Majid, M.I.A.; Amirul, A.A. Improved production of poly (3-hydroxybutyrate-co-4-hydroxbutyrate) copolymer using a combination of 1, 4-butanediol and $\gamma$-butyrolactone. World J. Microbiol. Biotechnol. 2010, 26, 743-746. [CrossRef]

34. Huong, K.H.; Teh, C.H.; Amirul, A.A. Microbial-based synthesis of highly elastomeric biodegradable poly (3-hydroxybutyrate-co-4-hydroxybutyrate) thermoplastic. Int. J. Biol. Macromol. 2017, 101, 983-995. [CrossRef]

35. Aziz, N.A.; Sipaut, C.S.; Abdullah, A.A.A. Improvement of the production of poly (3-hydroxybutyrate-co-3-hydroxyvalerate-co-4-hydroxybutyrate) terpolyester by manipulating the culture condition. J. Chem. Technol. Biotechnol. 2012, 87, 1607-1614. [CrossRef]

36. Ramachandran, H.; Amirul, A.A. Yellow-pigmented Cupriavidus sp., a novel bacterium capable of utilizing glycerine pitch for the sustainable production of P (3HB-co-4HB). J. Chem. Technol. Biotechnol. 2013, 88, 1030-1038. [CrossRef]

37. Hermann-Krauss, C.; Koller, M.; Muhr, A.; Fasl, H.; Stelzer, F.; Braunegg, G. Archaeal production of polyhydroxyalkanoate (PHA) co-and terpolyesters from biodiesel industry-derived by-products. Archaea 2013, 2013, 1-10. [CrossRef] [PubMed]

38. Koller, M.; Hesse, P.; Bona, R.; Kutschera, C.; Atlić, A.; Braunegg, G. Biosynthesis of high quality polyhydroxyalkanoate co-and terpolyesters for potential medical application by the archaeon Haloferax mediterranei. Macromol. Symp. 2007, 253, 33-39. [CrossRef]

39. Povolo, S.; Romanelli, M.G.; Basaglia, M.; Ilieva, V.I.; Corti, A.; Morelli, A.; Chiellini, E.; Casella, S. Polyhydroxyalkanoate biosynthesis by Hydrogenophaga pseudoflava DSM1034 from structurally unrelated carbon sources. New Biotechnol. 2013, 30, 629-634. [CrossRef]

40. Valentin, H.E.; Dennis, D. Production of poly (3-hydroxybutyrate-co-4-hydroxybutyrate) in recombinant Escherichia coli grown on glucose. J. Biotechnol. 1997, 58, 33-38. [CrossRef]

41. Li, Z.J.; Shi, Z.Y.; Jian, J.; Guo, Y.Y.; Wu, Q.; Chen, G.Q. Production of poly (3-hydroxybutyrate-co-4-hydroxybutyrate) from unrelated carbon sources by metabolically engineered Escherichia coli. Metab. Eng. 2010, 12, 352-359. [CrossRef]

42. Zhang, L.; Shi, Z.Y.; Wu, Q.; Chen, G.Q. Microbial production of 4-hydroxybutyrate, poly-4-hydroxybutyrate, and poly (3-hydroxybutyrate-co-4-hydroxybutyrate) by recombinant microorganisms. Appl. Microbiol. Biotechnol. 2009, 84, 909-916. [CrossRef] 
43. Kämpf, M.M.; Thöny-Meyer, L.; Ren, Q. Biosynthesis of poly (4-hydroxybutyrate) in recombinant Escherichia coli grown on glycerol is stimulated by propionic acid. Int. J. Biol. Macromol. 2014, 71, 8-13. [CrossRef] [PubMed]

44. Meng, D.C.; Shi, Z.Y.; Wu, L.P.; Zhou, Q.; Wu, Q.; Chen, J.C.; Chen, G.Q. Production and characterization of poly (3-hydroxypropionate-co-4-hydroxybutyrate) with fully controllable structures by recombinant Escherichia coli containing an engineered pathway. Metab. Eng. 2012, 14, 317-324. [CrossRef] [PubMed]

45. Chen, X.; Yin, J.; Ye, J.; Zhang, H.; Che, X.; Ma, Y.; Li, M.; Wu, L.P.; Chen, G.Q. Engineering Halomonas bluephagenesis TD01 for non-sterile production of poly (3-hydroxybutyrate-co-4-hydroxybutyrate). Bioresour. Technol. 2017, 244, 534-541. [CrossRef] [PubMed]

46. Shen, R.; Yin, J.; Ye, J.W.; Xiang, R.J.; Ning, Z.Y.; Huang, W.Z.; Chen, G.Q. Promoter engineering for enhanced P (3HB-co-4HB) production by Halomonas bluephagenesis. ACS Synth. Biol. 2018, 7, 1897-1906. [CrossRef] [PubMed]

47. Ye, J.; Hu, D.; Che, X.; Jiang, X.; Li, T.; Chen, J.; Zhang, H.M.; Chen, G.Q. Engineering of Halomonas bluephagenesis for low cost production of poly (3-hydroxybutyrate-co-4-hydroxybutyrate) from glucose. Metab. Eng. 2018, 47, 143-152. [CrossRef]

48. Ye, J.; Huang, W.; Wang, D.; Chen, F.; Yin, J.; Li, T.; Zhang, H.; Chen, G.Q. Pilot scale-up of poly (3-hydroxybutyrate-co-4-hydroxybutyrate) production by Halomonas bluephagenesis via cell growth adapted optimization process. Biotechnol. J. 2018, 13, 1800074. [CrossRef]

49. Cal, A.J.; Kibblewhite, R.E.; Sikkema, W.D.; Torres, L.F.; Hart-Cooper, W.M.; Orts, W.J.; Lee, C.C. Production of polyhydroxyalkanoate copolymers containing 4-hydroxybutyrate in engineered Bacillus megaterium. Int. J. Biol. Macromol. 2021, 168, 86-92. [CrossRef]

50. Zhang, S.; Liu, Y.; Bryant, D.A. Metabolic engineering of Synechococcus sp. PCC 7002 to produce poly-3-hydroxybutyrate and poly-3-hydroxybutyrate-co-4-hydroxybutyrate. Metab. Eng. 2015, 32, 174-183. [CrossRef]

51. Doi, Y.; Segawa, A.; Kunioka, M. Biosynthesis and characterization of poly (3-hydroxybutyrate-co-4-hydroxybutyrate) in Alcaligenes eutrophus. Int. J. Biol. Macromol. 1990, 12, 106-111. [CrossRef]

52. Saito, Y.; Doi, Y. Microbial synthesis and properties of poly (3-hydroxybutyrate-co-4-hydroxybutyrate) in Comamonas acidovorans. Int. J. Biol. Macromol. 1994, 16, 99-104. [CrossRef]

53. Kimura, H.; Yoshida, Y.; Doi, Y. Production of poly (3-hydroxybutyrate-co-4-hydroxybutyrate) by Pseudomonas acidovorans. Biotechnol. Lett. 1992, 14, 445-450. [CrossRef]

54. Tamer, I.M.; Moo-Young, M.; Chisti, Y. Disruption of Alcaligenes latus for recovery of poly ( $\beta$-hydroxybutyric acid): Comparison of high-pressure homogenization, bead milling, and chemically induced lysis. Ind. Eng. Chem. Res. 1998, 37, 1807-1814. [CrossRef]

55. Hiramitsu, M.; Koyama, N.; Doi, Y. Production of poly (3-hydroxybutyrate-co-4-hydroxybutyrate) by Alcaligenes latus. Biotechnol. Lett. 1993, 15, 461-464. [CrossRef]

56. Huong, K.H.; Kannusamy, S.; Lim, S.Y.H.; Amirul, A.A. Biosynthetic enhancement of single-stage poly (3-hydroxybutyrate-co-4-hydroxybutyrate) production by manipulating the substrate mixtures. J. Ind. Microbiol. Biotechnol. 2015, 42, 1291-1297. [CrossRef]

57. Kimura, H.; Ohura, T.; Matsumoto, T.; Ikarashi, T. Effective biosynthesis of poly (3-hydoxy-butyrate-co-4-hydroxybutyrate) with high 4-hydroxybutyrate fractions by Wautersia eutropha in the presence of $\alpha$-amino acids. Polym. Int. 2008, 57, 149-157. [CrossRef]

58. Madden, L.A.; Anderson, A.J.; Asrar, J.; Berger, P.; Garrett, P. Production and characterization of poly (3-hydroxybutyrate-co-3-hydroxyvalerate-co-4-hydroxybutyrate) synthesized by Ralstonia eutropha in fed-batch cultures. Polymer 2000, 41, 3499-3505. [CrossRef]

59. Abd Aziz, N.; Huong, K.H.; Sipaut, C.S.; Amirul, A.A. A fed-batch strategy to produce high poly (3-hydroxybutyrate-co-3-hydroxyvalerate-co-4-hydroxybutyrate) terpolymer yield with enhanced mechanical properties in bioreactor. Bioprocess Biosyst. Eng. 2017, 40, 1643-1656. [CrossRef]

60. Rao, U.; Sridhar, R.; Sehgal, P.K. Biosynthesis and biocompatibility of poly (3-hydroxybutyrate-co-4-hydroxybutyrate) produced by Cupriavidus necator from spent palm oil. Biochem. Eng. J. 2010, 49, 13-20. [CrossRef]

61. Hou, J.; Han, J.; Cai, L.; Zhou, J.; Lü, Y.; Jin, C.; Liu, J.; Xiang, H. Characterization of genes for chitin catabolism in Haloferax mediterranei. Appl. Microbiol. Biotechnol. 2014, 98, 1185-1194. [CrossRef] [PubMed]

62. Lin, L.; Chen, J.; Mitra, R.; Gao, Q.; Cheng, F.; Xu, T.; Zuo, Z.; Xiang, H.; Han, J. Optimising PHBV biopolymer production in haloarchaea via CRISPRi-mediated redirection of carbon flux. Commun. Biol. 2021, 4, 1-13. [CrossRef] [PubMed]

63. Han, J.; Zhang, F.; Hou, J.; Liu, X.; Li, M.; Liu, H.; Cai, L.; Zhang, B.; Chen, Y.; Zhou, J.; et al. Complete genome sequence of the metabolically versatile halophilic archaeon Haloferax mediterranei, a poly (3-hydroxybutyrate-co-3-hydroxyvalerate) producer. J. Bacteriol. 2012, 194, 4463-4464. [CrossRef]

64. Vigneswari, S.; Vijaya, S.; Majid, M.I.A.; Sudesh, K.; Sipaut, C.S.; Azizan, M.N.M.; Amirul, A.A. Enhanced production of poly (3-hydroxybutyrate-co-4-hydroxybutyrate) copolymer with manipulated variables and its properties. J. Ind. Microbiol. Biotechnol. 2009, 36, 547-556. [CrossRef] [PubMed]

65. Huong, K.H.; Azuraini, M.J.; Aziz, N.A.; Amirul, A.A.A. Pilot scale production of poly (3-hydroxybutyrate-co-4-hydroxybutyrate) biopolymers with high molecular weight and elastomeric properties. J. Biosci. Bioeng. 2017, 124, 76-83. [CrossRef]

66. Norhafini, H.; Huong, K.H.; Amirul, A.A. High PHA density fed-batch cultivation strategies for 4HB-rich P (3HB-co-4HB) copolymer production by transformant Cupriavidus malaysiensis USMAA1020. Int. J. Biol. Macromol. 2019, 125, 1024-1032. [CrossRef] [PubMed] 
67. Azira, T.F.; Nursolehah, A.A.; Norhayati, Y.; Majid, M.I.A.; Amirul, A.A. Biosynthesis of Poly (3-hydroxybutyrate-co-3-hydroxyvalerate-co-4-hydroxybutyrate) terpolymer by Cupriavidus sp. USMAA2-4 through two-step cultivation process. World J. Microbiol. Biotechnol. 2011, 27, 2287-2295. [CrossRef]

68. Kucera, D.; Novackova, I.; Pernicova, I.; Sedlacek, P.; Obruca, S. Biotechnological production of poly (3-hydroxybutyrate-co-4-hydroxybutyrate-co-3-hydroxyvalerate) terpolymer by Cupriavidus sp. DSM 19379. Bioengineering 2019, 6, 74. [CrossRef]

69. Kim, J.S.; Lee, B.H.; Kim, B.S. Production of poly (3-hydroxybutyrate-co-4-hydroxybutyrate) by Ralstonia eutropha. Biochem. Eng. J. 2005, 23, 169-174. [CrossRef]

70. Chanprateep, S.; Katakura, Y.; Visetkoop, S.; Shimizu, H.; Kulpreecha, S.; Shioya, S. Characterization of new isolated Ralstonia eutropha strain A-04 and kinetic study of biodegradable copolyester poly (3-hydroxybutyrate-co-4-hydroxybutyrate) production. J. Ind. Microbiol. Biotechnol. 2008, 35, 1205-1215. [CrossRef]

71. Raposo, R.S.; de Almeida, M.C.M.; da Fonseca, M.M.R.; Cesário, M.T. Feeding strategies for tuning poly (3-hydroxybutyrate-co-4-hydroxybutyrate) monomeric composition and productivity using Burkholderia sacchari. Int. J. Biol. Macromol. 2017, 105, 825-833. [CrossRef] [PubMed]

72. Hein, S.; Söhling, B.; Gottschalk, G.; Steinbüchel, A. Biosynthesis of poly (4-hydroxybutyric acid) by recombinant strains of Escherichia coli. FEMS Microbiol. Lett. 1997, 153, 411-418. [CrossRef]

73. Tripathi, L.; Wu, L.P.; Meng, D.; Chen, J.; Chen, G.Q. Biosynthesis and characterization of diblock copolymer of P (3-hydroxypropionate)-block-P (4-hydroxybutyrate) from recombinant Escherichia coli. Biomacromolecules 2013, 14, 862-870. [CrossRef] [PubMed]

74. Zhou, Q.; Shi, Z.Y.; Meng, D.C.; Wu, Q.; Chen, J.C.; Chen, G.Q. Production of 3-hydroxypropionate homopolymer and poly (3-hydroxypropionate-co-4-hydroxybutyrate) copolymer by recombinant Escherichia coli. Metab. Eng. 2011, 13, 777-785. [CrossRef]

75. Pries, A.N.D.R.E.A.S.; Priefert, H.O.R.S.T.; Krüger, N.; Steinbüchel, A. Identification and characterization of two Alcaligenes eutrophus gene loci relevant to the poly (beta-hydroxybutyric acid)-leaky phenotype which exhibit homology to ptsH and ptsI of Escherichia coli. J. Bacteriol. 1991, 173, 5843-5853. [CrossRef]

76. Syafiq, I.M.; Huong, K.H.; Shantini, K.; Vigneswari, S.; Abd Aziz, N.; Amirul, A.A.A.; Bhubalan, K. Synthesis of high 4-hydroxybutyrate copolymer by Cupriavidus sp. transformants using one-stage cultivation and mixed precursor substrates strategy. Enzyme Microb. Technol. 2017, 98, 1-8. [CrossRef] [PubMed]

77. Lau, N.S.; Sudesh, K. Revelation of the ability of Burkholderi a sp. USM (JCM 15050) PHA synthase to polymerize 4-hydroxybutyrate monomer. AMB Express 2012, 2, 1-9. [CrossRef]

78. Al-Kaddo, K.B.; Mohamad, F.; Murugan, P.; Tan, J.S.; Sudesh, K.; Samian, M.R. Production of P (3HB-co-4HB) copolymer with high 4HB molar fraction by Burkholderia contaminans Kad1 PHA synthase. Biochem. Eng. J. 2020, 153, 107394. [CrossRef]

79. Hori, K.; Kaneko, M.; Tanji, Y.; Xing, X.H.; Unno, H. Construction of self-disruptive Bacillus megaterium in response to substrate exhaustion for polyhydroxybutyrate production. Appl. Microbiol. Biotechnol. 2002, 59, 211-216. [PubMed]

80. Valappil, S.P.; Peiris, D.; Langley, G.J.; Herniman, J.M.; Boccaccini, A.R.; Bucke, C.; Roy, I. Polyhydroxyalkanoate (PHA) biosynthesis from structurally unrelated carbon sources by a newly characterized Bacillus spp. J. Biotechnol. 2007, 127, 475-487. [CrossRef]

81. Hu, D.; Chung, A.L.; Wu, L.P.; Zhang, X.; Wu, Q.; Chen, J.C.; Chen, G.Q. Biosynthesis and characterization of polyhydroxyalkanoate block copolymer P3HB-b-P4HB. Biomacromolecules 2011, 12, 3166-3173. [CrossRef] [PubMed] 